\title{
LIFE FACTORS AFFECTING DEPRESSION AND BURDEN IN AMYOTROPHIC LATERAL SCLEROSIS CAREGIVERS
}

by

\section{Kristen Qutub}

B.S., University of Richmond, 2011

\author{
Submitted to the Graduate Faculty of \\ the Graduate School of Public Health in partial fulfillment \\ of the requirements for the degree of \\ Master of Science
}

University of Pittsburgh

2013 
UNIVERSITY OF PITTSBURGH

Graduate School of Public Health

This thesis was presented

by

\section{Kristen Qutub}

It was defended on

March 29, 2013

and approved by

Thesis Advisor:

David Lacomis, M.D.

Professor, Department of Neurology

School of Medicine, University of Pittsburgh

Committee Member:

Robert Ferrante, Ph.D., M.S.

Professor, Department of Neurological Surgery

School of Medicine, University of Pittsburgh

Committee Member:

Stephen Albert, Ph.D.

Professor and Chair, Behavioral and Community Health

Graduate School of Public Health, University of Pittsburgh

Committee Member:

Eleanor Feingold, Ph. D.

Professor, Human Genetics

Graduate School of Public Health, University of Pittsburgh 
Copyright (C) by Kristen Qutub

2013 


\title{
LIFE FACTORS AFFECTING DEPRESSION AND BURDEN IN AMYOTROPHIC LATERAL SCLEROSIS CAREGIVERS
}

\author{
Kristen Qutub, M.S.
}

University of Pittsburgh, 2013

Amyotrophic lateral sclerosis (ALS), similar to other degenerative disorders, can be especially challenging to cope with for friends and relatives, as well as for the patient. Often, as the disease progresses, a full-time caregiver is necessary to provide consistent support and care. While previous studies have proposed that the majority of caregivers of ALS patients are not clinically depressed, it has been observed that some depression symptoms are still present and that ALS caregivers may continue to experience changes in temperament. The purpose of this study was to determine which factor or factors in a caregiver's life contribute to the caregiver experiencing a decline in his/her perceived quality of life and/or an increased overall feeling of burden. The five factors assessed were financial status, social support, patient disease progression, employment, and religious affiliation. Caregivers were evaluated using the Beck Depression Inventory Revised survey, Zarit-Burden Interview, and a demographic survey. This information was used to assess overall mental and emotional stress exhibited by the caregivers. Age, sex, financial burden, religious affiliation, and the daily hours spent both with the patient and actively caregiving were all shown to have an association with Beck Depression Inventory scores, indicating that these elements are significant risk factors for depression symptoms. Both age and the daily hours spent caregiving were also associated with higher Zarit-Burden Interview scores. Additionally, employment status and social support were found to be associated with Zarit-Burden Interview scores. Social support was the only factor in the caregivers' lives found 
to be protective, as caregivers citing adequate social support systems had lower Zarit-Burden Interview scores. The other factors associated with either depression or burden were all found to be risk factors. There was no statistical significant association between a caregiver's BDI or ZBI score and patient progression based on the patient's ALS-FRS score. Using this information, new interventions and programs can be established, with the goal to prevent caregiver depression and alleviate caregiver burden as much as possible. Establishing a caregiver-specific support group is a logical first-step that may be highly beneficial for ALS caregivers. The information collected in this study is also important from a public health standpoint because these results will enable healthcare providers to better understand the stress and burden of caregivers, allowing more appropriate interventions and resources to be implemented and offered throughout the course of the disease. It will also aid in the more general understanding of the mental health effects of caring for dependents with degenerative diseases. 
TABLE OF CONTENTS

ACKNOWLEDGEMENTS _....................................................................................................... XI

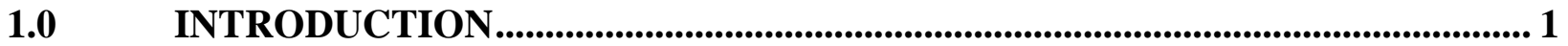

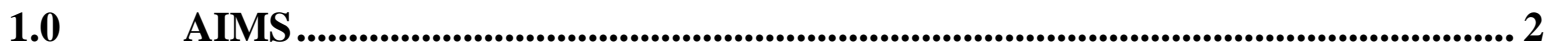

1.1 BACKGROUND AND SIGNIFICANCE ...................................................... 2

1.1.1 Caregivers.................................................................................................................. 4

1.1.2 Caregiver depression ............................................................................................... 6

1.1.3 Burden ................................................................................................................. 7

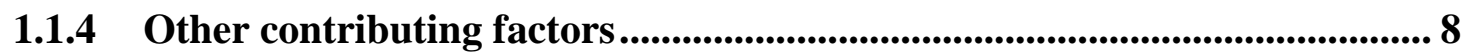

1.1.5 Significance................................................................................................................ 9

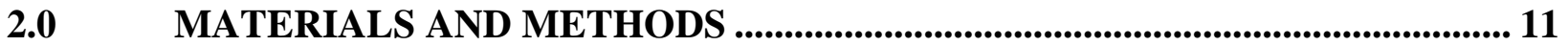

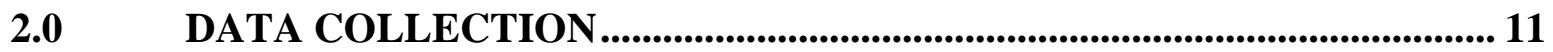

2.0.1 Patient population...................................................................................................... 11

2.0.2 Patient recruitment and informed consent ..................................................... 12

2.0.3 Sample................................................................................................................. 13

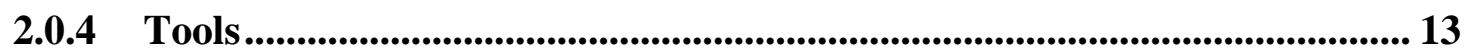

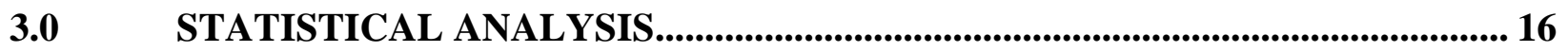

3.0.1 Caregiver participants ........................................................................................... 16

3.0.2 Caregiver depression and burden ..................................................................... 18 
3.0.3 Patient progression ....................................................................................... 20

3.0.4 Statistically significant other life factors ...................................................... 22

3.0.5 Non-statistically significant other life factors ................................................. 32

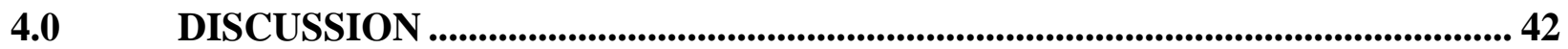

4.0.1 Implications for ALS caregivers ................................................................. 42

4.0.2 Public Health Significance .................................................................................. 44

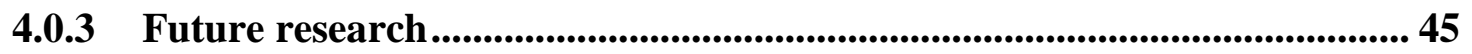

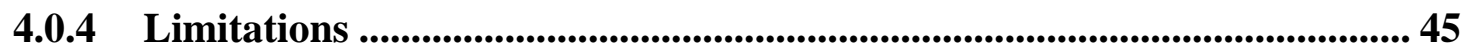

CONCLUSION.................................................................................................................. 47

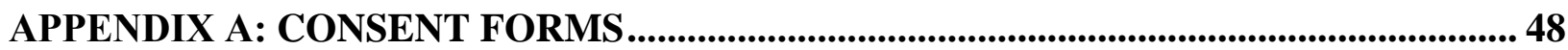

APPENDIX B: DEMOGRAPHIC SURVEY ................................................................................. 62

BIBLIOGRAPHY 


\section{LIST OF TABLES}

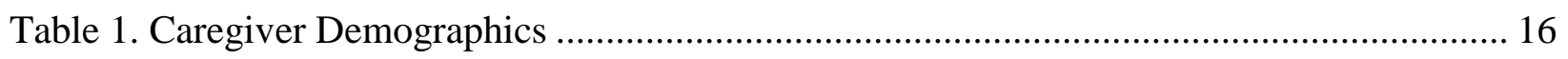

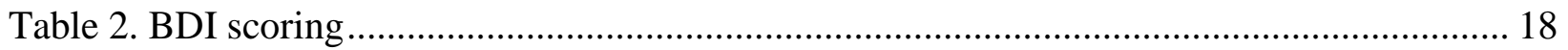

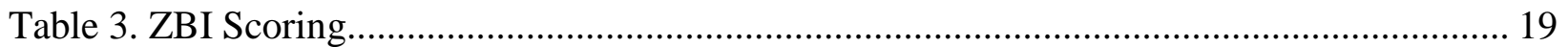




\section{LIST OF FIGURES}

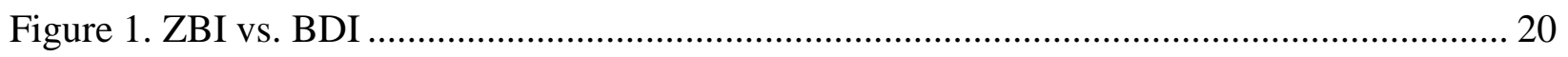

Figure 2. BDI vs. ALSFRS score..................................................................................... 21

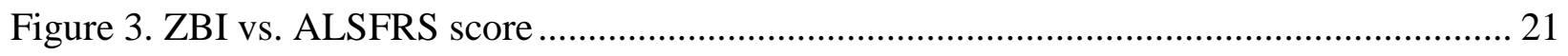

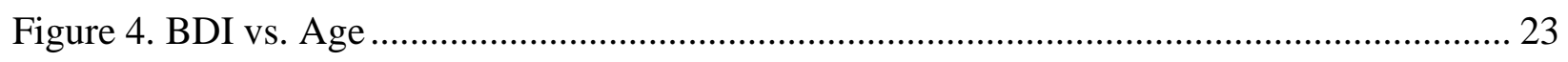

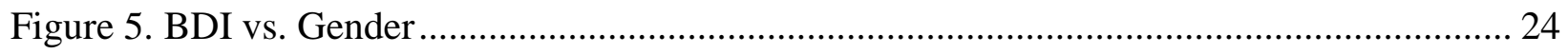

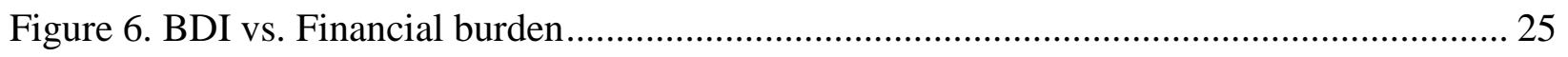

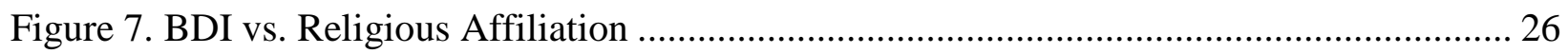

Figure 8. BDI vs. Daily hours spent with the patient................................................................... 27

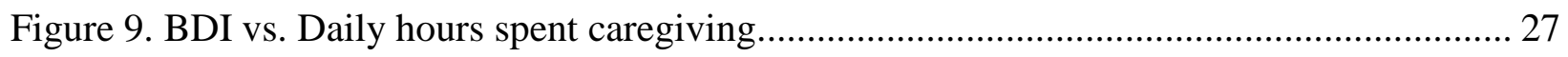

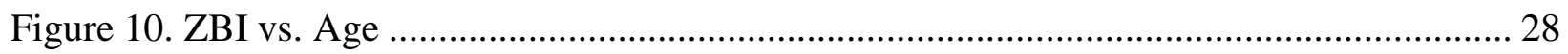

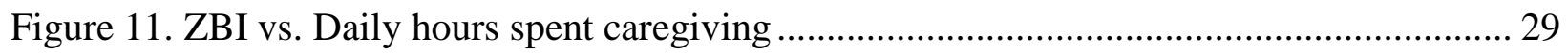

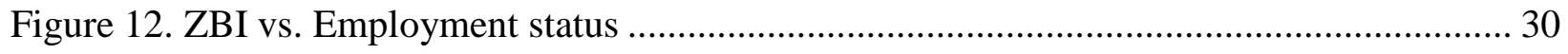

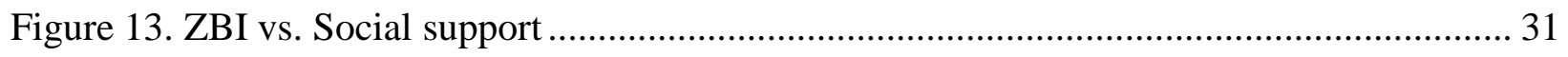

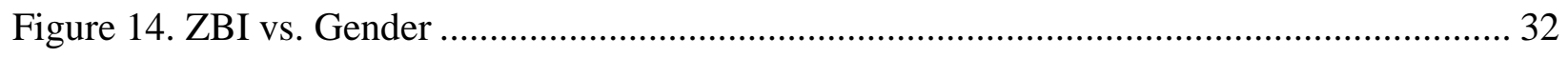

Figure 15. ZBI vs. Religious Affiliation............................................................................ 32

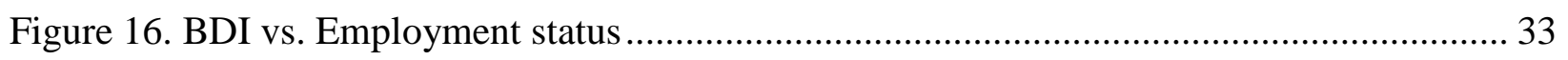

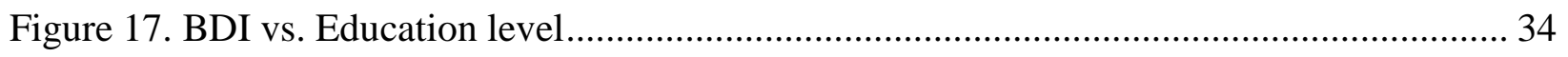

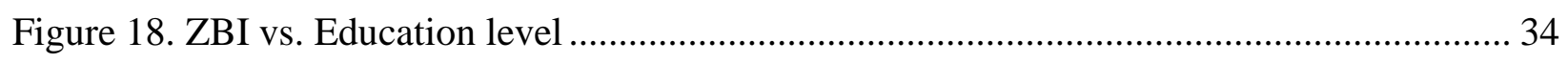




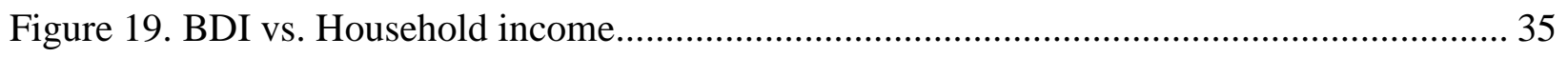

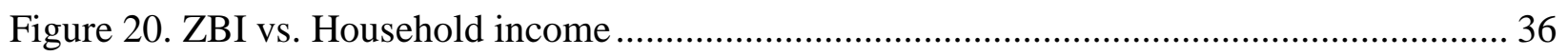

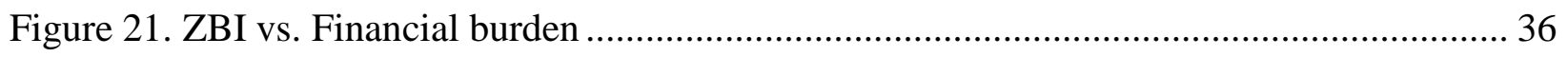

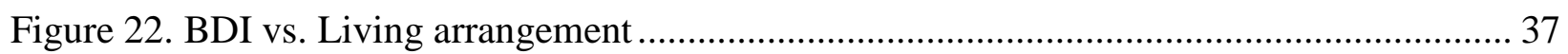

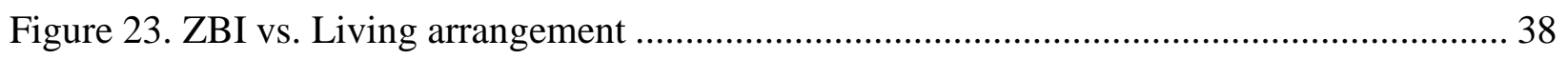

Figure 24. BDI vs. Hours of outside help received per week ............................................... 39

Figure 25. ZBI vs. Hours of outside help received per week .............................................. 39

Figure 26. BDI vs. Religion as a part of daily life .......................................................... 40

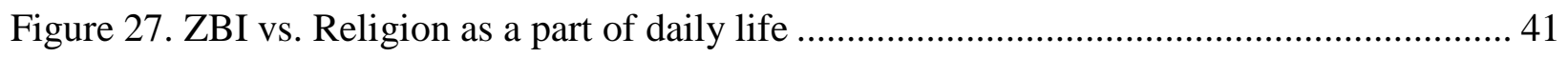

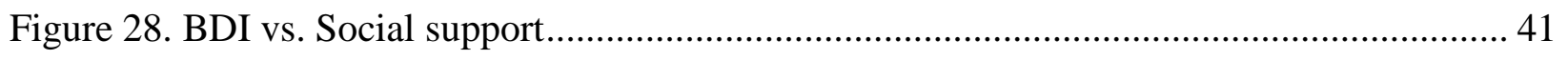




\section{ACKNOWLEDGEMENTS}

First and foremost, I would like to thank Dr. David Lacomis for giving me the opportunity to be a part of this exciting research project. He allowed me to further explore my interest in psychosocial issues and ALS research, and I greatly appreciate his support and guidance. A warm thanks to Dr. Sasa Zivkovic, Becky Molczan, and Sandy Fera. They patiently provided me with the materials I needed and answered all of my ALS-related questions. I would also like to thank the other members of my thesis committee, Dr. Steven Albert, Dr. Robert Ferrante, and Dr. Eleanor Feingold. Their expertise and help has been invaluable throughout this research project.

I would like to extend my gratitude to my program directors Betsy Gettig and Dr. Robin Grubs. Their guidance and support have aided me throughout this journey the past two years. I would like to acknowledge my genetic counseling classmates for their continued pep talks, help, and support. I look forward to continuing to keep in touch in the future, as we all embark out into the real world.

It is also important to acknowledge all of the ALS families that participated in this study. I was incredibly lucky to work with such inspiring and loving individuals, who were incredibly eager to become involved in research. Clinic days are always very busy and I appreciate the extra time that these patients and caregivers volunteered. I will always treasure the experiences and time that I spent with our ALS community. 
I would like to thank my family and boyfriend for their unconditional love and support. I have always been able to fall back on them, finding a listening ear and helpful suggestions. Thank you for always believing in me and continuing to encourage me when things became difficult.

Lastly, and most importantly, I would like to dedicate this thesis work to Ben Gill. His fight motivated me to become involved in ALS research. Watching his determination and courage as he fought ALS has always been a point of inspiration and he continues to be missed every single day. Thank you to his wife, Sally, and daughter, Lori, for showing me the strength and love of ALS families, empowering me to spread that understanding and compassion to the families I worked with for this study. 


\subsection{INTRODUCTION}

Amyotrophic Lateral Sclerosis (ALS) is a devastating progressive neurodegenerative disease that leads to a fatal loss of striated muscle movement in an affected individual. From the time of the onset of symptoms, disease progression can last months or years. In all cases, however, advancement of ALS leads to an individual requiring assistance to perform everyday tasks. With the continued loss of muscle function, eating, bathing, walking, speaking, and dressing all become increasingly difficult. At the end stages of life, individuals with ALS need full time care. This care is most often provided by a spouse, child, other relative, or close friend. Paid caregivers can be difficult to find or families may be unable to financially afford paid help. Therefore, an unpaid caregiver is faced with the daily tasks of caring for the patient. Frequently, in addition to providing care and support, the caregiver is also faced with other responsibilities including paying bills, cleaning, laundry, taking care of children, etc. With all of these outside stressors and the undeniable stress associated with caring for a sick loved one, caregiver health is directly affected and often caregivers report a decline in their quality of life.

This study was undertaken to ascertain whether or not there was a specific factor or factors in caregivers' lives that led to a significant increase or decrease in overall depression or burden. The main goal was to establish a contributing factor to increased depression or burden in order to initiate an intervention for our caregivers, preserving, as best we can, their quality of life. In order to achieve this goal, caregivers were asked to complete surveys which assessed 
their depression level, overall burden, and demographic information. The survey results were analyzed using a number of statistical tests, evaluating the association between caregiver depression and burden levels with their other demographic factors and personal routine. The following thesis outlines the study protocol, results, and conclusions.

\subsection{AIMS}

The first aim of this study is to assess the depression level, burden, and general quality of life of ALS caregivers. The second aim of this study is to determine which of five possible factors (finances, patient progression, employment, social support, and religious affiliation) contribute the most to the caregivers' burden and/or depression symptoms. The data presented and discussed here provides the foundation for better anticipating the needs of ALS caregivers, in order to establish support systems and intervention programs. It is hypothesized that there will be a factor or factors in the caregivers' lives that are correlated with their depression or burden, therefore increasing stress and anxiety and decreasing their overall quality of life.

\subsection{BACKGROUND AND SIGNIFICANCE}

Amyotrophic lateral sclerosis (ALS), also publically known as Lou Gehrig's disease, is a progressive neurodegenerative disorder that leads to deterioration and death of the motor neurons. Motor neurons are nerve cells located in the brain (upper motor neurons) and spinal cord (lower motor neurons). Their degeneration prohibits impulse transmission and leads to 
improper functioning of the voluntary muscles, which causes weakness, atrophy, and fasciculations. With ALS, muscle impairment progresses until the ability to control striated muscle movements is entirely lost. Often individuals with ALS die due to respiratory failure when the muscles in the chest and diaphragm are unable to function.

According to statistics from the National Institute of Health, about 30,000 individuals have ALS in the United States. As many as 5,000 people are diagnosed with the condition each year, breaking down to about 15 new cases per day (National Institute of Neurological Disorders and Stroke, 2012). A relatively common neuromuscular disorder, ALS appears to affect all races and ethnicities but is seen most often in Caucasians. According to the ALS Association, the ALS CARE database records indicate that $93 \%$ of cases registered are Caucasian and $60 \%$ are male (ALS Association, 2010). The age of onset is typically between the ages of 40 to 70 with a median age of diagnosis of 55, but there is a wide variability for the onset of symptoms. Most cases of ALS occur sporadically with only 5-10\% of cases determined to be familial or have clear patterns of inheritance (National Institute of Neurological Disorders and Stroke, 2012).

Diagnosing ALS is very difficult. Symptoms can be mild and overlooked or mimic the symptoms of other conditions. The diagnosis of ALS is often a diagnosis of exclusions. Individuals with ALS must have symptoms or signs of both upper and lower motor neuron damage that cannot be attributed to another cause. There is not a single test available to definitively diagnose ALS; instead a clinical diagnosis through a neurological evaluation combined with excluding other possibilities is the standard method of diagnosis. Electromyography and nerve conduction velocity tests are used to support or refute the diagnosis. Since the diagnosis of ALS is not always clear, a person may have numerous testing 
and unnecessary procedures or surgeries and still go years without a correct diagnosis. This is incredibly frustrating for both the individual and their loved ones.

Early symptoms may include stiffness, twitching, or cramping. General muscle weakness, slurred or nasal speech, and difficulty chewing or swallowing are also early signs of the disease. Different individuals notice varying symptoms. However, regardless of where the disease first presents, the condition progresses to greater muscle weakness and atrophy. Moving becomes difficult and the individual can experience dysphagia, dysarthria, spasticity, hyperreflexia, and muscle cramps. This advancement of symptoms attributes to the individual no longer being able to stand, walk, or use his/her extremities. Daily tasks such as getting out bed, dressing, bathing, and eating become extremely difficult or impossible for the individual. With this progression, a caregiver is a necessity (Mayo Clinic, 2011; National Institute of Health, 2013).

\subsubsection{Caregivers}

Since the disease inevitably disables an individual, even with the best care and treatment available, caregiving is an extremely important aspect of the disease. The Family Caregiver Alliance defines a caregiver as "a person who provides assistance to someone else who is, in some degree, incapacitated and needs help.” As of 2010, there were 52 million informal and family caregivers in the United States, providing care to someone aged 20 or older who is sick or disabled (Coughlin, 2010). Unpaid family caregivers are the largest source of long-term care services in the United States.

Of the millions of caregivers, about 59-75\% of caregivers are female. Previous reports have also observed that female caregivers, under the age of 75 , spend $50 \%$ more time providing care than their male caregiver counterparts and often perform the more strenuous tasks of 
caregiving, such as bathing, toileting, and dressing (Health and Human Services, 1998). In the United States, the majority of caregivers are between 35-64 years of age and most caregivers are the older child/children or spouse of the ill individual. In a national sampling of caregivers who lived with their care recipients, spouses accounted for $62 \%$ of primary caregivers while the adult children comprised $26 \%$ of the total (Kennedy, 1997). While many caregivers live with their care recipient or live nearby, 5.1 to 7 million caregivers live an hour or more away (Metropolitan Life, 2010; Wagner, 1997). In addition, 48\% of caregivers report working full time and $11 \%$ report working part time (Belden, 2004).

With the amount of time spent caregiving on a daily basis, it is astounding to see the high number of caregivers who retain their employment status. Approximately $20 \%$ of caregivers provide more than 40 hours of care a week (Belden, 2004). While about $28 \%$ of the caregivers providing over 40 hours of care a week are over the age of 65 and may be retired or fall into the part time employment category, it is essential to remember that often the older caregivers are caring for older individuals who may require more intensive care and have more significant illnesses or disabilities. For an overall estimate of the time dedicated to caregiving in the United States, a study by the California Caregiver Resource Center estimated that the average number of hours their caregivers provided support was 81 hours per week (Department of Mental Health, 2005). With the extensive number of caregivers in the United States, a number that is continuing to increase, caregiving is a prevalent occurrence and the health of caregivers is a serious issue of importance. 


\subsubsection{Caregiver depression}

As ALS progresses, the caregiver has less and less time to adjust to the changes, both physical and emotional, occurring in both the patient and within themselves. With the extensive time commitment and a significant emotional burden, much research has been done to evaluate caregiver stress and depression. Study results, however, have been incredibly varied in this area. Several studies have identified that ALS caregivers experience depression. Rabkin et al. (2009) conducted a study in which, at baseline, $13 \%$ of caregivers had major depression and $10 \%$ of caregivers registered minor depression (Rabkin, 2009). Ganzini et al. (1998) found even more evidence, concluding that $22,24 \%$ of the caregivers in their study, were determined to be clinically depressed (Ganzini, 1998). Gauthier et al. (2007) also noted a significant increase in overall burden and depression in caregivers (Gauthier, 2007). Interestingly, Chio et al. (2005) reported that caregiver burden and depression increased with the care recipient's ALS progression (Chio, 2005). Pagnini et al (2010) also noted that the patient's severity of disease directly correlated with caregiver burden and depression (Pagnini, 2010). While these studies all agree that many ALS caregivers experience depression, other research has produced conflicting results.

There have been multiple studies that have shown inconsistent results comparing caregiver depression and patient disease progression. Data collected from the Beck Depression Inventory and other self-reporting questionnaires has shown, on average, that most caregivers experience very few depression symptoms (Mockford, Jenkinson, \& Fitzpatrick, 2006). Although not excluding depression entirely, Trail et al. (2003) found that few caregivers registered any depression, with other caregivers only having mild depression symptoms (Trail, 2003). Shultz et al. (1995) reported similar data, recognizing a significant minority of spousal 
caregivers develop depression when caring for a person with a long-term progressive illness (Shultz, 1995). Rabkin et al. (2009) also found that only a small minority of caregivers of ALS patients registered depression and that this number remained stable regardless of patient progression (Rabkin, 2009). Contrary to previous research, their study found no association between patient progression and caregiver depression (Rabkin, 2009). While it is clear that depression symptoms are clearly present in some ALS caregivers, one cannot determinately state that all caregivers are depressed. However, it is reasonable to conclude, both from research and common sense, that all ALS caregivers have some degree of burden with the arduous task of caregiving.

\subsubsection{Burden}

Caregiver burden is a term that is used in reference to the intense stress and anxiety experienced by caregivers while they assist and tend to an individual with an illness. There are a plethora of stressors experienced by caregivers. There is a degree of physicality involved in providing care including lifting the individual, toileting, bathing, and dressing. Additionally, many household chores often become the responsibility of the caregiver. This occurs frequently if the caregiver is a spouse. Bills, cleaning, cooking, laundry, and a multitude of other tasks fall upon the caregiver. Lillo et al. (2012) describe caregiver burden as “a complex construct involving the caregivers’ feelings about the emotional/physical health, social life, and financial status that they suffer as a result of caring for their relatives” (Lillo, 2012). While burden is not differentiated as a psychological diagnosis, this perpetual pressure and stress associated with caregiving has a substantial impact on caregiver health. 


\subsubsection{Other contributing factors}

That there is a strain associated with caregiving for an ALS patient is undeniable. However, there is more than an emotional strain. Caregiving in ALS also requires a high physical effort. As the disease progresses, the individual with ALS requires substantial assistance to move, bathe, and toilet. This is especially challenging for older caregivers or caregivers with their own disability. Recognizing that advancing age or physical exertion are only some of the possible contributing factors, there is a surprisingly small amount of research that has been conducted regarding other factors and their influence on caregiver burden.

Calvo et al. (2011) reported that caregivers’ quality of life was related to their private religiousness (Calvo, 2011). Religion was positively correlated with ALS caregivers' quality of life and overall satisfaction and was, in fact, the second most important predictor of caregiver quality of life after anxiety. This suggested that religion and spirituality may help caregivers cope with their current situations, providing a more positive attitude. Another study evaluated social support systems and caregiver burden. It was reported that people in older age groups, over the age of 60, tended to have consistent support systems throughout their caregiving period. Conversely, younger caregivers, under the age of 60, noted more fluctuations in their personal relationships and declines in social support as caregiving intensity increased (Ray, 2005). This advocates the need for interventions and programs for younger caregivers, to ensure that the younger population of caregivers receives continued support throughout their period of providing care. However, this study was conducted in Australia and social support was defined broadly. It is uncertain whether this data would extrapolate to the United States and there are no other research studies to supplement this conclusion. 


\subsubsection{Significance}

Some studies have touched upon the fact that there are numerous other factors within a caregiver's life and that these other outside factors may play a role in increased caregiver burden and a decreased quality of life. As Rabkin et al. (2009) noted, distress among caregivers is not universal (Rabkin, 2009). Since not all caregivers, or even half of ALS caregivers, register a diagnosis of clinical depression, it leads one to question as to what factors in a caregiver's life are contributing or protecting from stress and perceived burden. Previous research has kept caregiver stressors as general concepts, evaluating time-dependent burden versus developmental burden versus emotional burden. Levels of physical and social burden have also been evaluated (Gauthier, 2007); (Chio, 2005). Mockford et al. (2006) probed a little deeper, reporting that the maintenance of social support and a positive attitude may improve caregivers' physical health (Mockford et al., 2006) . Likewise, Goldstein et al. (2006) noted that psychological factors appeared to be important determinants for both short and long term mental health (Goldstein, 2006). However, no one has yet delved deeper into those categories, to really examine the cause of the developmental or emotional burden. To find the specific source of that burden so that it can be targeted with intervening measures.

Research, up to this point, has found no correlations between age, gender, education level, income, or available outside help and caregiver depression (Rabkin, 2009). No correlations have been seen between caregiver depression and the caregiver's age, gender, or participation in support groups either (Chio, 2005). Yet, even though these external factors showed no correlation to depression thus far, no one has offered data examining whether these external factors are directly related to caregiver burden. Burden and depression are often closely associated, but their underlying causes and development can be distinctly different. Oyebode et 
al. (2013) have observed that caregivers seem to inhibit their grief in order to appear strong for their loved ones (Oyebode, Smith, \& Morrison, 2013). Therefore, a more thorough understanding of the causes behind caregiver burden would help to provide more appropriate emotional support.

This study aimed to thoroughly observe multiple aspects of the ALS caregivers' lives to examine the association between those external factors and depression, but also those external factors and caregiver burden. Finding a distinct relationship between other factors in a caregiver's life and increased burden could allow one to better understand caregiver stress and establish therapies and programs in order to best alleviate or diminish those stressors. Specifically, this study analyzed caregivers' employment status, financial burden, social support, religiousness, and the disease progression of the patient. While ALS caregivers are often strong and resilient individuals, it is important that they receive the best care and help possible as well. 


\subsection{MATERIALS AND METHODS}

Kristen Qutub, B.S. conducted the surveys under Institutional Review Board approval from the University of Pittsburgh (PRO12020466).

\subsection{DATA COLLECTION}

\subsubsection{Patient population}

The patient population consisted of caregivers of ALS patients that attend the ALS Multidisciplinary Clinic at the University of Pittsburgh Medical Center. Inclusion criteria were that caregivers had to be over the age of 18 , be unpaid, and spend a minimum of at least three hours of time a day caring for the patient. Care could include, but was not limited to, bathing, dressing, feeding, transporting, and providing emotional support. In a case where a patient had multiple caregivers meeting the study requirements, only the caregiver who spent the greatest amount of time caring for the patient was invited to participate. Exclusion criteria were caregivers under the age of 18 or any paid caregivers, including caregivers who worked at a nursing facility or provided in-home care. Caregivers caring for patients with ALS and dementia were not excluded. 


\subsubsection{Patient recruitment and informed consent}

Most participant recruitment and informed consent was obtained in the ALS Multidisciplinary Clinic in the Kaufman Medical Building in Pittsburgh, Pennsylvania. The researcher approached those that expressed interest in participating and informed consent was obtained after explanation of the protocol, study aims, risks, and benefits. Some participants were recruited by telephone and consent forms were mailed and returned. All recruitment was with compliance of IRB protocol number PRO12020466. Patients were recruited by a genetic counseling intern or a neurologist.

Informed consent involved a discussion of the background and aims of the research project as well as participation requirements, participant rights, and the risks and benefits associated with the study. After discussion of the research study, participants were given adequate time to read the consent form and to ask any further questions. Consenting caregivers provided a signature, printed name, and date on the last page of the consent form.

While the ALS caregivers were the participants in this study, the patients were also asked to provide consent. Patients were asked to provide permission for their ALS clinic notes to be released for use in this study. Patients were informed that their participation was voluntary and it was explained how the clinic notes would be used without breaching confidentiality. Patients were also given adequate time to read the consent form and to ask any additional questions. Consenting patients provided a signature, printed name, and date on the last page of the consent form. In some cases, surveys were mailed out to caregivers who were unable to attend clinic or who had not been present with their care recipient. These patients were first consented by phone and then additionally mailed hard copies of the consent form and the surveys to sign, fill out, and 
return. No surveys were obtained without the written consent of both the caregiver and the patient.

\subsubsection{Sample}

We studied 50 caregivers, all recruited from the ALS Multidisciplinary clinic population at University of Pittsburgh Medical Center in Pittsburgh, Pennsylvania. Of the caregivers that were eligible to participate in the study, 2 refused participation and 11 surveys were not returned. The 11 surveys not returned were surveys that had been mailed to caregivers. All surveys completed in clinic were returned to the researcher. Caregivers were asked to participate in the study between May 2012 and February 2013.

\subsubsection{Tools}

\section{Beck Depression Inventory}

The Beck Depression Inventory (BDI-II) is a 21 item questionnaire designed as a self-rating scale to measure changes in mood related to depression. For the purposes of this study, the most recently revised BDI version was used. The original BDI was published in 1961, with a revision in 1978; with the most recent modification, the BDI-II, being accepted in 1996. The BDI-II was created to identify the presence and severity of depression symptoms, as listed in the American Psychiatric Association’s Diagnostic and Statistical Manual of Mental Disorders Fourth Edition (DSM-IV; 1994) rather than establishing a clinical diagnosis of depression. The BDI-II is a widely used tool used in a variety of forums and practices (Beck \& Steer, 1984; Beck, Ward, Mendelson, Mock, \& Erbaugh, 1961). 
The BDI-II asks questions regarding sleep habits, appetite, body image, weight fluctuations, work ability, and energy levels. Each question allows the participant to rate themselves on a four-point scale ranging from 0-4. At the completion of the BDI-II, the researcher totals the overall score. Scoring of 0-9 indicates minimal depression, 10-16 indicates mild depression, 17-29 indicates moderate depression, and 30-63 indicates severe depression.

BDI has been used for decades to identify and assess depression symptoms and, to date, has reported to be highly reliable regardless of the population. This questionnaire has a highcoefficient alpha (0.92), its construct validity has been established, and it is believed to accurately differentiate between depressed and non-depressed patients.

\section{Zarit-Burden Interview}

The Zarit-Burden Interview (ZBI) is a 22 item questionnaire designed as a self-reporting mechanism to establish burden associated with behavioral impairments and the home-care situation. Each question is worded to focus on the affective response of the caregiver.

The ZBI was first published in 1980 as a 29 item questionnaire. It has since been revised and, in 1983, a 22 item questionnaire was created. For the purposes of this study, the newer and shorter version of the ZBI was used. Questions on the ZBI ask about emotions of the caregiver, including stress, embarrassment, fear, and guilt. It also asks about the caregiver's perceptions of his/her social life and overall health. For each of the 22 questions, a scoring system from 0 (never) to 4 (nearly always present) is available. The researcher can then add the final score which will range from 0 (low burden) to 88 (high burden). A study, conducted in 2000 by Hérbert, Bravo, and Préville, evaluated 213 caregivers from the Canadian Study of Health and Aging. The results of this study proposed that the ZBI had good internal consistency reliability, 
with a high-coefficient alpha of 0.92 , in a variety of populations. An additional study by Seng et al. in 2010 calculated an alpha coefficient of 0.93 , further concluding that the ZBI was a valid and reliable tool instrument in measuring the burden of caregivers (Bedard et al., 2001).

\section{ALSFRS-R}

The ALS Functional Rating Scale-Revised (ALSFRS-R) is a twelve item assessment, measuring the disease severity in a patient. The scale focuses on rating an individual's fine motor skills, gross motor skills, and bulbar and respiratory function. Each of the twelve items is scored from 0 to 4, for an overall possible total of 48. Lower ALSFRS-R score indicate greater disability and an estimated shorter survival period.

\section{Demographic Survey}

The final tool was a demographic assessment (Appendix B). General demographic information, including gender and age, was collected. Additionally, the demographic data sheet addressed questions regarded to the five factors analyzed in this study. Questions about employment status, education level, annual household income, hours caregiving, living situations, available social support, and religiosity were all included. 


\subsection{STATISTICAL ANALYSIS}

\subsubsection{Caregiver participants}

Fifty caregivers were recruited for this analysis. Demographics are listed below in Table 1 . The first column indicates the factor examined; the second column states the average score or response for that specific life factor. The p-values for both BDI and ZBI are listed for each aspect of the caregiver's life. Statistical analysis was performed using t-tests, regression analysis, and ANOVA and the test used is differentiated in the last column.

Table 1. Caregiver Demographics

\begin{tabular}{|c|c|c|c|c|}
\hline & Means & BDI p value & ZBI p value & Statistical analysis \\
\hline BDI & 9.55 & \multirow{2}{*}{\multicolumn{2}{|c|}{$<\mathbf{0 . 0 0 0 1}^{1}$}} & Regression \\
\hline ZBI & 23.56 & & & Regression \\
\hline ALS-FRS & 34.10 & 0.3758 & 0.0632 & Regression \\
\hline $\mathrm{Age}^{2}$ & 60.80 years & 0.0359 & 0.0049 & Regression \\
\hline Gender $^{3}$ & $\begin{array}{l}34 \% \text { male } \\
66 \% \text { female }\end{array}$ & 0.0391 & 0.0588 & t-test \\
\hline Employment status ${ }^{4}$ & $\begin{array}{c}\text { 36\% employed } \\
64 \% \text { unemployed/retired }\end{array}$ & 0.7072 & 0.0399 & t-test \\
\hline Education level & $\begin{array}{c}36 \% \text { HS diploma } \\
30 \% \text { some college } \\
30 \% \text { college degree } \\
2 \% \text { graduate degree }\end{array}$ & 0.3263 & 0.9488 & ANOVA \\
\hline Household income & $\begin{array}{c}11 \%<\$ 20,000 \\
30 \% \$ 20,001-40,000 \\
25 \% \$ 40,001-60,000 \\
14 \% \$ 60,001-80,000 \\
7 \% \$ 80,001-100,000\end{array}$ & 0.0931 & 0.3335 & Regression \\
\hline
\end{tabular}




\begin{tabular}{|c|c|c|c|c|}
\hline & $\begin{array}{c}\% \$ 100,001-120,000 \\
2 \% \$ 120,001-140,000 \\
7 \%>\$ 140,001 \\
\end{array}$ & & & \\
\hline Financial burden & $\begin{array}{c}\text { 8\% Significant burden } \\
\text { 24\% Small burden } \\
68 \% \text { No burden } \\
\end{array}$ & 0.0296 & 0.3082 & $\begin{array}{l}\text { ANOVA } \\
\text { ANOVA }\end{array}$ \\
\hline Living arrangement & $\begin{array}{c}\text { 67\% living with } \\
\text { spouse/partner only }\end{array}$ & 0.8288 & 0.3470 & t-test \\
\hline $\begin{array}{l}\text { Daily hours spent } \\
\text { with patient }\end{array}$ & 16.80 & 0.0058 & 0.6941 & Regression \\
\hline $\begin{array}{l}\text { Daily hours caring } \\
\text { for patient }\end{array}$ & 8.22 & 0.0071 & 0.0479 & Regression \\
\hline $\begin{array}{l}\text { Percentage of daily } \\
\text { time spent } \\
\text { caregiving }\end{array}$ & $7.86 \%$ & 0.2023 & 0.0534 & Regression \\
\hline $\begin{array}{l}\text { Outside help } \\
\text { received }\end{array}$ & $\begin{array}{l}37 \% \text { yes } \\
63 \% \text { no }\end{array}$ & 0.4230 & 0.4061 & Regression \\
\hline $\begin{array}{l}\text { Hours of outside } \\
\text { help received per } \\
\text { week }\end{array}$ & 7.71 & 0.9471 & 0.4098 & Regression \\
\hline $\begin{array}{l}\text { Adequate social } \\
\text { support }\end{array}$ & $\begin{array}{l}79 \% \text { receive adequate } \\
\text { social support }\end{array}$ & 0.1100 & $0.0210^{5}$ & t-test \\
\hline Religious affiliation & $\begin{array}{c}\text { 44\% Catholic } \\
\text { 33\% Protestant } \\
\text { 17\% Other Christian } \\
\text { 6\% Atheist/Agnostic/Other }\end{array}$ & $0.0055^{6}$ & 0.0531 & ANOVA \\
\hline $\begin{array}{l}\text { Religion as a part } \\
\text { of daily life }\end{array}$ & $\begin{array}{l}54 \% \text { believe religion is a } \\
\text { part of their daily life } \\
46 \% \text { do not consider } \\
\text { religion a part of their daily } \\
\text { life }\end{array}$ & 0.6185 & 0.3307 & t-test \\
\hline Religiosity & $\begin{array}{c}25 \% \text { not religious } \\
17 \% \text { a little religious } \\
37 \% \text { religious } \\
21 \% \text { very religious }\end{array}$ & 0.9750 & 0.6758 & Regression \\
\hline
\end{tabular}

1. When compared, BDI was found to be positively correlated with ZBI at $\mathrm{p}=<0.0001$

2. Younger ages corresponded with higher BDI and ZBI scores

3. Females had higher BDI scores

4. Caregivers retaining employment had greater ZBI scores

5. Caregivers reporting adequate social support had lower ZBI scores

6. Catholic and Protestant caregivers had lower BDI scores than caregivers reporting other Christian. Since only three individuals reported atheist, agnostic, and other, these categories were not included in the statistical analysis. 


\subsubsection{Caregiver depression and burden}

Consistent with other previous research, this study found that ALS caregivers did not register diagnoses of clinical depression (Mockford et al., 2006; Shultz, 1995; Trail, 2003). Of the 50 caregivers surveyed, no one scored in the severe depression range, 30 - 63, using the Beck Depression Inventory Revised self-reported survey. Ten individuals, 20\%, did register moderate depression, a score of 17 - 29. Twenty-five percent of our caregivers, twelve individuals, reported BDI scores of 10-16, indicating mild depression. The remaining 27 caregivers, 55\%, had a BDI score of $0-9$, or no depression. The average BDI score was 9.55, a score indicating none or low depression. However, despite the fact that the majority of caregivers indicated no depression or mild depression, it is important to note that there was a range in BDI scores from 0 to 29. This range of scores again signifies the relevance of this study as it revisits the question of why some caregivers experience depression symptoms while over half of the caregivers surveyed reported no depression.

Table 2. BDI scoring

\begin{tabular}{|l|l|}
\hline BDI score & \% of caregivers scoring in this range \\
\hline $0-9$ (none) & $55 \%$ \\
\hline $10-16$ (mild) & $25 \%$ \\
\hline $17-29$ (moderate) & $20 \%$ \\
\hline $30-63$ (severe) & $0 \%$ \\
\hline
\end{tabular}


Caregivers also recorded a large range of scores on the Zarit Burden Interview, demonstrating a wide spectrum of perceived burden. Scores ranged from 3 to 52 . Twenty-five caregivers, 50\%, had a ZBI score from 0 - 20 which correlates with little or no burden. Mild to moderate burden was characterized by a score of $21-40$ and 15 of our caregivers, $30 \%$, were in this category. Ten of the caregivers, $20 \%$, achieved a score of $41-60$, which is associated with moderate to severe burden. None of the 50 caregivers surveyed registered severe burden at a ZBI score between 61 and 88. The average ZBI score of 23.56, however, identifies that most caregivers suffer some degree of burden. This finding also supports previous research. As expected, ALS caregivers do not meet the clinical criteria for depression, but still experience significant daily levels of burden within their lives. This study also showed a significant association between a caregiver's BDI and ZBI score (Figure 1). As a caregiver's BDI score increased, his/her ZBI score increased in tandem. This result is important as it indicates that as a caregiver's burden increases, his/her risk for a clinical depression diagnosis is also increased. While research has shown that ALS caregivers are not often in the clinical depression category, this result could change if caregiver burden levels rise or are not relieved.

Table 3. ZBI Scoring

\begin{tabular}{|l|l|}
\hline ZBI score & $\%$ of caregivers scoring in this range \\
\hline $0-20$ (little to none) & $50 \%$ \\
\hline $21-40$ (mild to moderate) & $30 \%$ \\
\hline $41-60$ (moderate to severe) & $20 \%$ \\
\hline $61-88$ (severe) & $0 \%$ \\
\hline
\end{tabular}




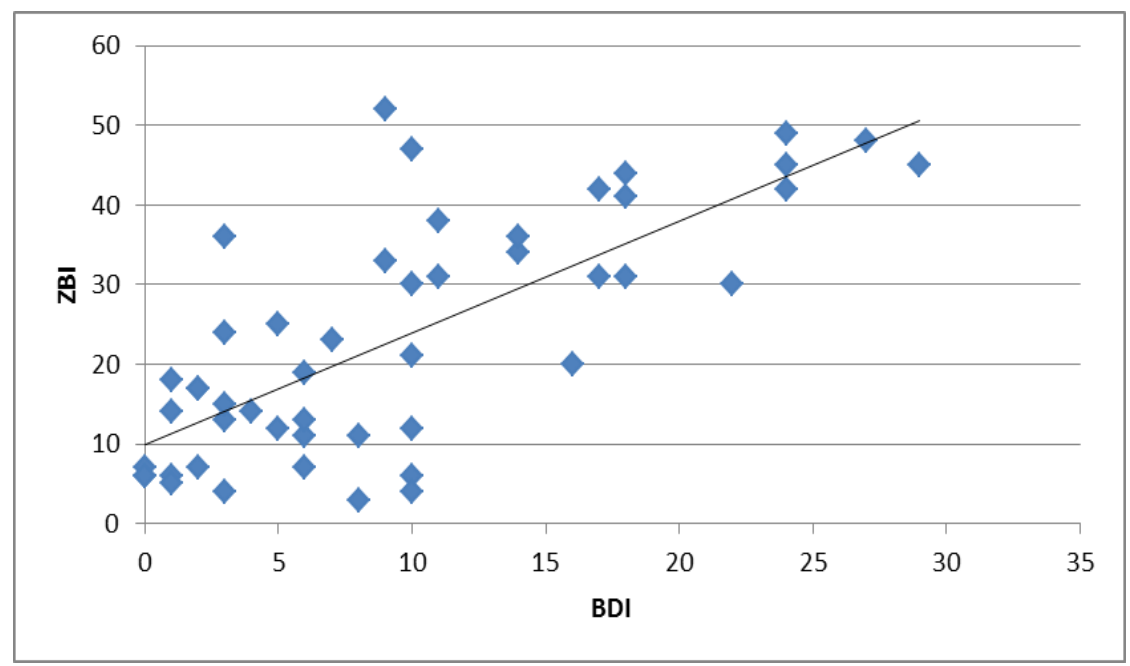

Figure 1. ZBI vs. BDI

\subsubsection{Patient progression}

When investigating the caregivers' depression level and overall burden, there was no statistically significant association between these numbers and the patient's disease status (Figure 2; Figure 3). Only one patient had clinically diagnosed dementia along with an ALS diagnosis. The caregiver of this patient, however, did not display high BDI or ZBI scores. Whether the patient was newly diagnosed and required little physical help or whether the patient was very weak and needed consistent care, did not predict the depression symptoms or burden rating of the caregivers. These data were consistent with Rabkin et al. (2009), who found no relationship between the patient’s progression and caregiver depression (Rabkin, 2009). Likewise, Lillo et al. (2012) noted that the patient's physical state was non-contributory to caregiver depression or burden (Lillo, 2012). These findings conflict with other research conducted by Chio et al. (2005) and Pagnini et al. (2010) who both reported seeing an increase in caregiver depression proportional to the patient's disease severity. While the collected data for patient progression versus caregiver depression is just as inconsistent as the data obtained regarding ALS caregiver 
depression in general, defining this association was not our only focus. This study looked beyond just the disease severity and caregiving duties, delving deeper into the other facets of the caregivers' lives.

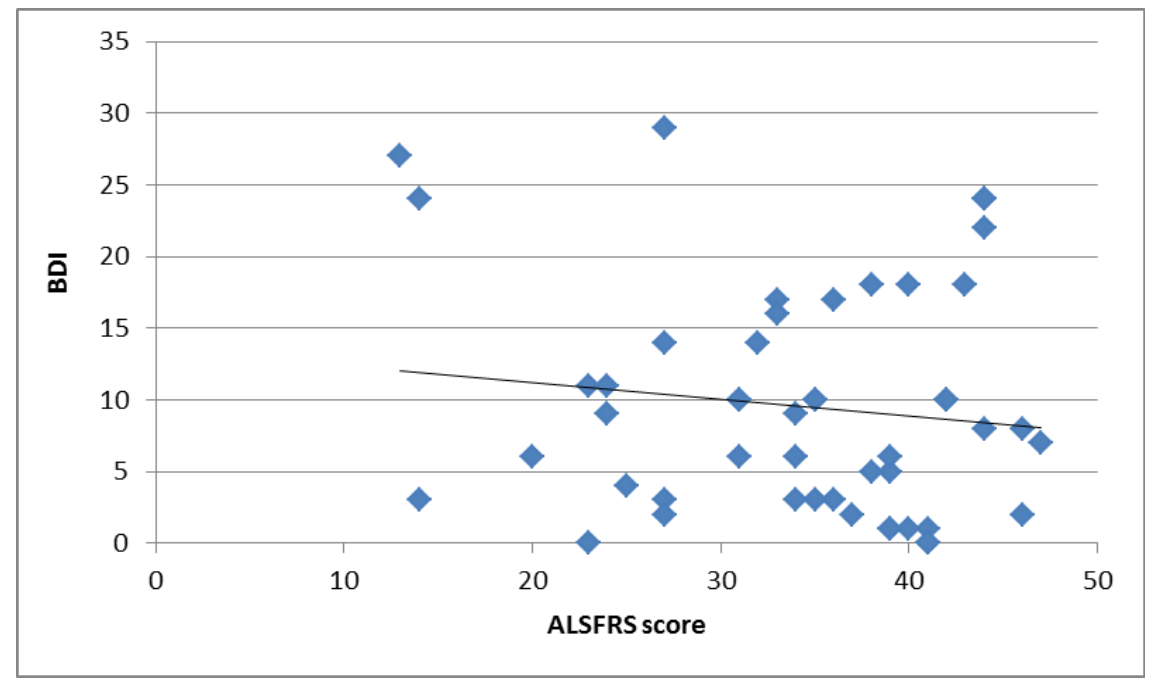

Figure 2. BDI vs. ALSFRS score

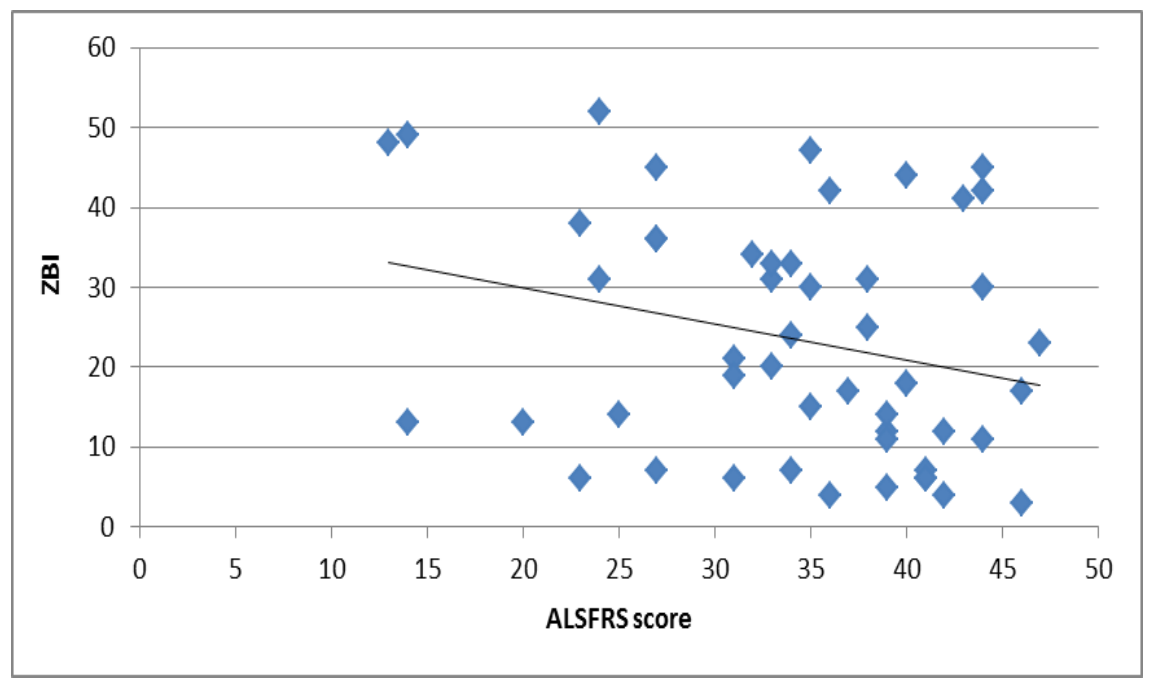

Figure 3. ZBI vs. ALSFRS score 


\subsubsection{Statistically significant other life factors}

This study primarily analyzed five main factors in a caregiver's life: financial burden, religiousness, social support, employment, and patient progression. In addition, some supplementary demographic information was obtained, including age, gender, and the living arrangement of the caregiver. With respect to the BDI, there was a significant association with six aspects of the caregivers' lives. In terms of demographics, caregiver’s depression scores were associated with age (Figure 4) and sex (Figure 5). With an average caregiver age around 61, caregivers showed greater depression at younger ages with a p-value of 0.0359. Depression levels, according to the BDI results, showed a drop in depression symptoms starting in the mid 50 age group. Older caregivers had less depression symptoms. This could be occurring for variety of reasons, as older caregivers have more experience with handling distress and illness and a fatal disease is less surprising at a later age of onset. For younger caregivers, having to care for a loved one with ALS is often completely unexpected. This may be the first time a younger individual may be faced with a difficult, life-changing event and his/her coping skills may be poor. Additionally, younger caregivers may still have younger children at home, still be establishing their careers, and not have the financial resources of an older couple. Ray (2005) noted that younger caregivers had less stable support systems and more fluctuations in their personal relationships, creating further instability for the young caregiver (Ray, 2005). 


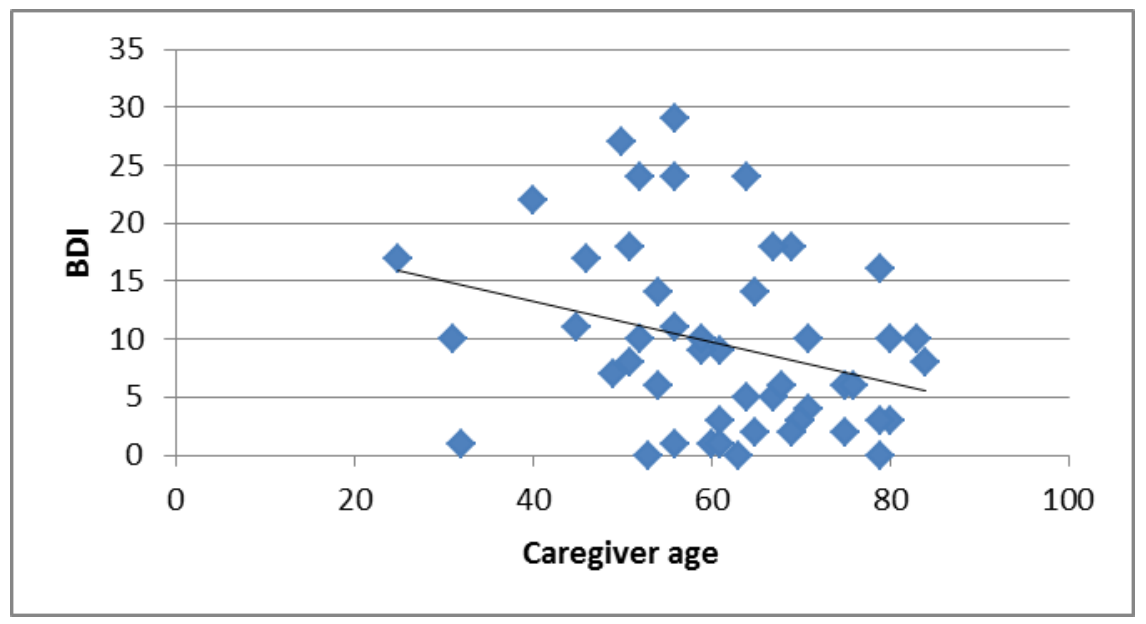

Figure 4. BDI vs. Age

Depression scores were also associated with the caregiver's sex with a p-value of 0.0391 . Female caregivers had higher BDI scores than their male counterparts. In this study, 66\% of the caregivers were females and 34\% were males. Of the 16 male caregivers surveyed, only one caregiver registered a score higher than 10. Conversely, many female caregivers scored much higher and there was a larger range of scores among female caregivers as seen in Figure 5. In previous literature, no relationships between caregiver depression and caregiver age or gender had been established (Chio, 2005). Yet, these statistics correlate with data stating that female caregivers often spend more time caregiving than male caregivers, as well as perform the most strenuous tasks of caregiving (Health and Human Services, 1998). This acquired information about the demographic correlations with depression scoring is interesting and made it even more important to look for further contributing factors in caregivers’ lives. 


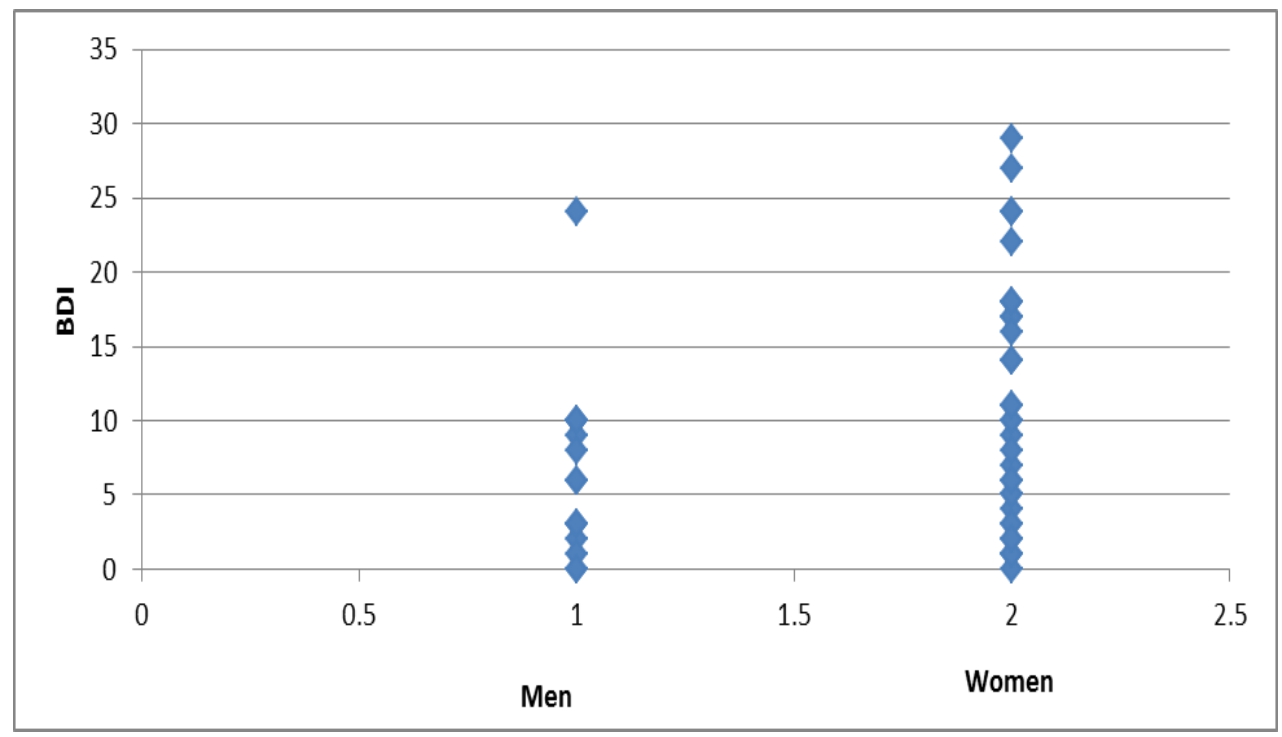

Figure 5. BDI vs. Gender

In asking about their finances, caregivers were instructed to record whether or not their finances were adequate or whether they felt a small or significant financial burden while caring for an individual with ALS. Of the caregivers surveyed, 68\% thought their finances were adequate and had no burden, 24\% reported a small financial burden, and 8\% said their financial strain was significant. In comparing all three financial categories using an ANOVA, there was a statistically significant difference in BDI, with a calculated p-value of 0.0296. Using a t-test, there was also a statistical significance in comparing caregivers who felt they had a small financial burden to caregivers who felt they had adequate finances, with a p-value of 0.0397 (Figure 6). Similarly, there was a statistical significance in comparing caregivers who felt they had a significant financial burden to caregivers who felt they had adequate finances with a pvalue of 0.0101 . According to these results, caregiver BDI scores are higher with financial burden. Having adequate financial resources correlates to less depression symptoms. 


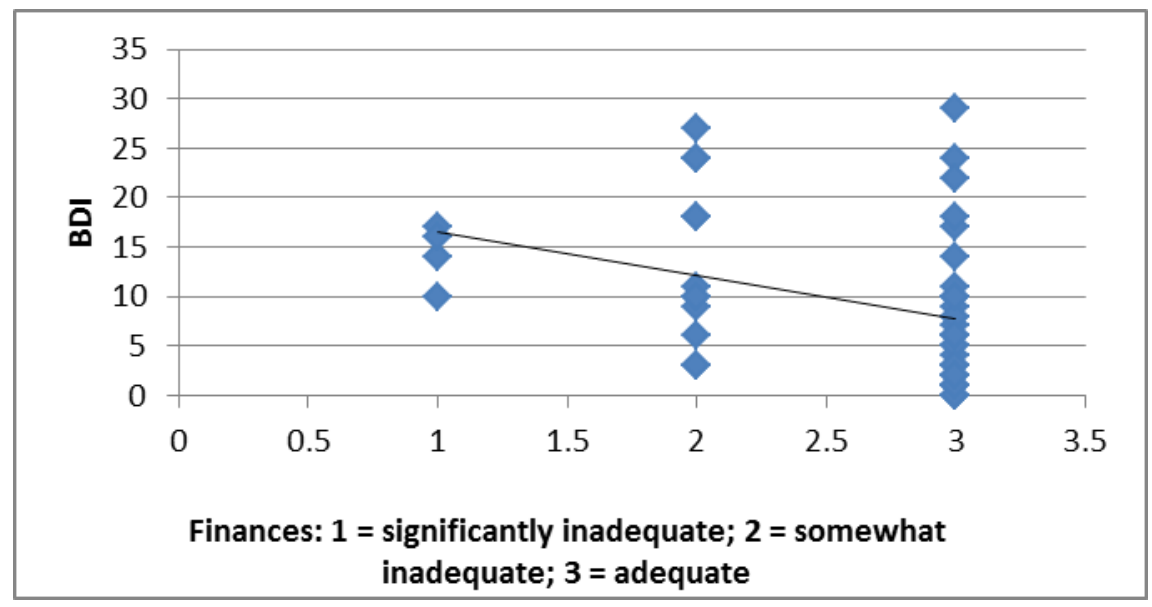

Figure 6. BDI vs. Financial burden

A stable financial situation equates to a lesser score on the BDI. Interestingly, religious affiliation also showed a significant difference with a p-value of 0.0055 (Figure 7). Caregivers were asked what religious group they identified with most. Caregivers identified only with the following denominations: 44\% Catholic, 33\% Protestant, 17\% Other Christian, 2\% agnostic, 2\% atheist, or $2 \%$ other. In Figure 7 below, Catholics are listed as 1, Protestants as 2, Other Christian as 3 , agnostics as 4, atheists as 5 , and other as 6 . Since only one caregiver chose each of the latter three options, those caregivers were not used in the statistical analysis quoted above. Rather, the comparison was focused between Catholic, Protestant, and Other Christian caregivers. In conducting additional t-tests, it was found that Catholic and Protestant caregivers had less depression symptoms than caregivers who identified as Other Christian, with p-values of 0.0169 and 0.0076 respectively. While analysis of overall religiosity, including religiousness and the inclusion of religion in daily life, was not significant, it is interesting that religious denomination contributed to decreased depression. This could be contributed to better coping skills associated with specific religious beliefs or a stronger religious community to provide support. This finding supports the research conducted by Calvo et al. (2011), associating caregiver quality of life to caregiver religiousness (Calvo, 2011). Although Calvo et al. (2011) did not study caregiver 
depression directly, instead focusing on quality of life, this study has shown that depression levels and overall burden are related, suggesting the two are not exclusive.

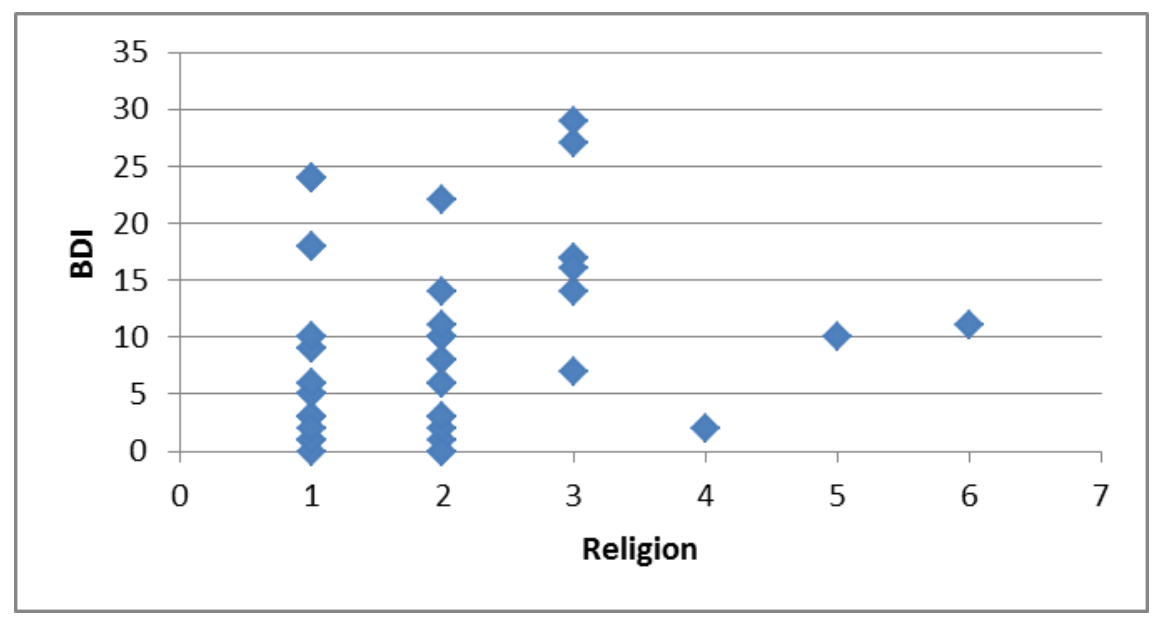

Figure 7. BDI vs. Religious Affiliation

The final significant statistical result associated with caregiver depression is the analysis of the daily total hours a caregiver spent with a patient (Figure 8) and the daily total hours actively caregiving (Figure 9). The average daily hours a caregiver spent with a patient is 16.8 . This number, however, is somewhat deceptive since it is only the mean. Most caregivers reported either spending a few hours a day with the patient or being with the patient 24 hours a day consistently. Most caregivers are spouses and spend all of their time with their loved one since they live together. Even if they are not providing constant care, there is little or no relief from the situation and very limited time to oneself. Additionally, the average number of hours spent providing assistance to the patient was 8.22 per day. This equates to a full day of corporate work and is a significant amount of time when it is taken into consideration that many of these caregivers are still employed outside the home, are also raising children, and/or must still complete necessary household chores. Previous statistics have agreed with this study, indicating that about $20 \%$ of caregivers put in a minimum of 40 hours of time weekly (Belden, 2004). This substantial time commitment directly affects caregiver depression, as a clear association is seen 
both between the number of hours caregivers spend with the patient (p-value 0.0058) and the number of hours caregivers are providing care (p-value 0.0071) and caregiver depression. Caregivers spending more time with the patient have increased depression symptoms. Interestingly, however, while it is undeniable that both the daily hours spent with the patient and hours spent caregiving are significant, the total percentage of daily time spent caregiving was not when compared with BDI, with a p-value of 0.2023 . This result could be skewed by caregivers who are spouses, who spend 24 hours a day with their loved one but do not have to provide many hours of care yet.

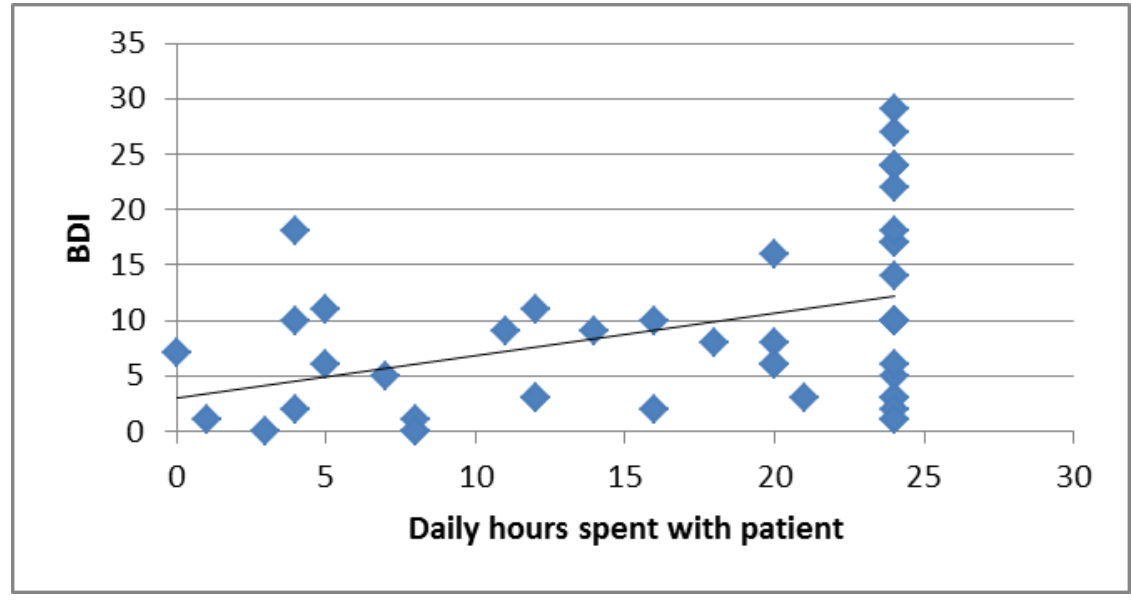

Figure 8. BDI vs. Daily hours spent with the patient

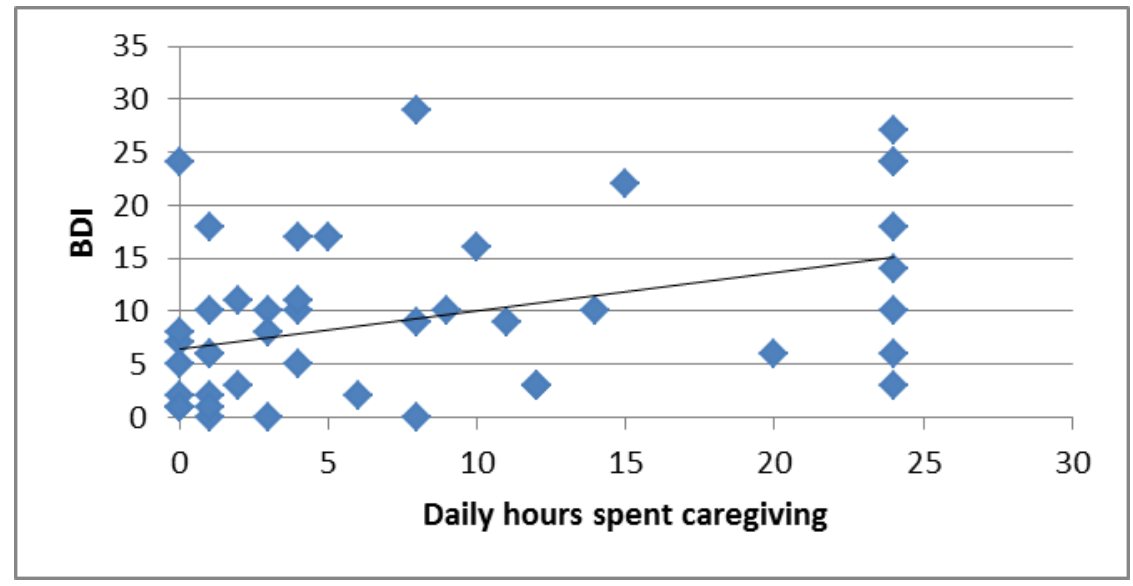

Figure 9. BDI vs. Daily hours spent caregiving 
According to the collected data in this study, age, gender, religious affiliation, financial burden, daily hours caregiving, and daily hours spent with the patient were all found to have a statistically significant association with caregiver depression. In analyzing our original five factors in the caregivers' lives compared to caregiver burden, some of the above correlations remained significant while others fluctuated. Age was one demographic feature that still proved to be a significant risk factor (Figure 10). Like the BDI scoring, younger caregivers scored higher on the ZBI, indicating higher degrees of burden with a p-value of 0.0049 . This trend could be for many of the same reasons listed above when comparing the BDI scores. Younger caregivers may have more outside elements to juggle, including a new career, small children, a new mortgage, student loans, and/or less established social support networks. It is also a possibility that older caregivers already have a considerable burden in their lives associated with the detriments of old age. For these older caregivers, a loved one being diagnosed with ALS may be less unexpected since it is often not the first health issue the individual experiences. Consequently, older caregivers may characterize their current burden differently if they were already dealing with an established level of stress.

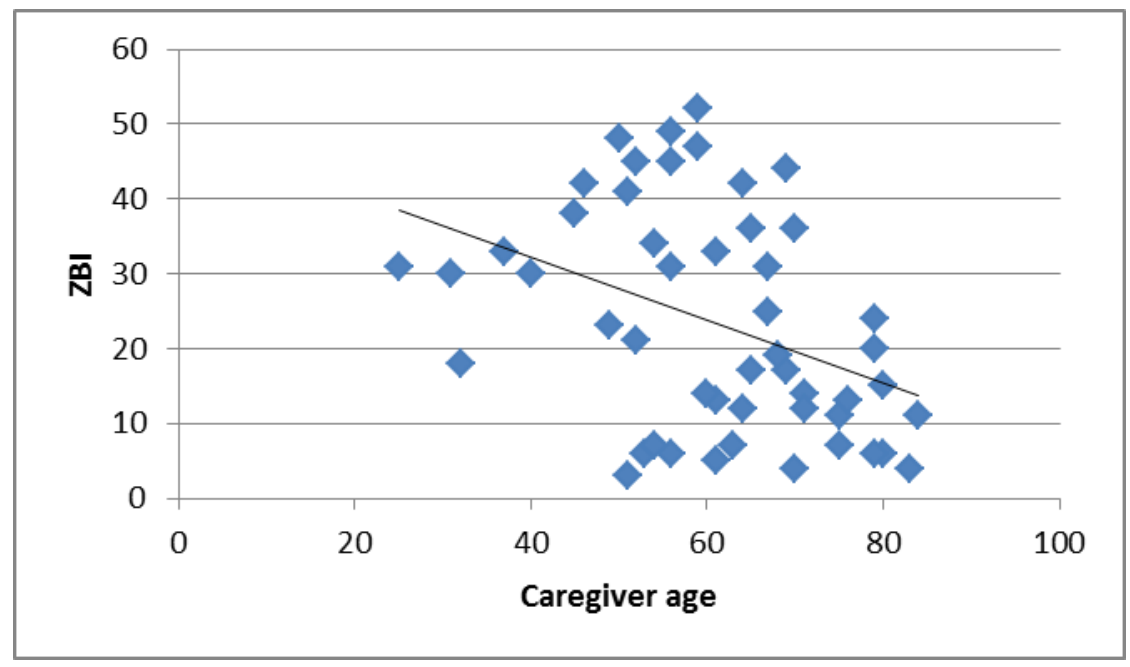

Figure 10. ZBI vs. Age 
Also, in accordance with the BDI results, the daily hours caregiving for the patient directly affected caregiver burden (Figure 11). When compared with the ZBI, there was a p-value of 0.0479 . Interestingly, the daily hours spent with the patient was not statistically significant with a p-value of 0.6941 . Rather, it was the actual daily provision of care that increased caregiver burden and anxiety. While it was not statistically significant, the percentage of daily time spent caregiving still showed a relationship when compared with ZBI, with a p-value of 0.0534 . This percentage was the number of hours spent providing care per day divided by the total number of daily hours spent with the patient. The type of caregiving performed was not specified, so the hours devoted to caregiving could be simple tasks such as cutting food or extensive help such as bathing. Regardless, these caregivers dedicate a sizable amount of every day to the relative or friend they care for. As shown by this data, this time obligation places a considerable strain on the caregivers.

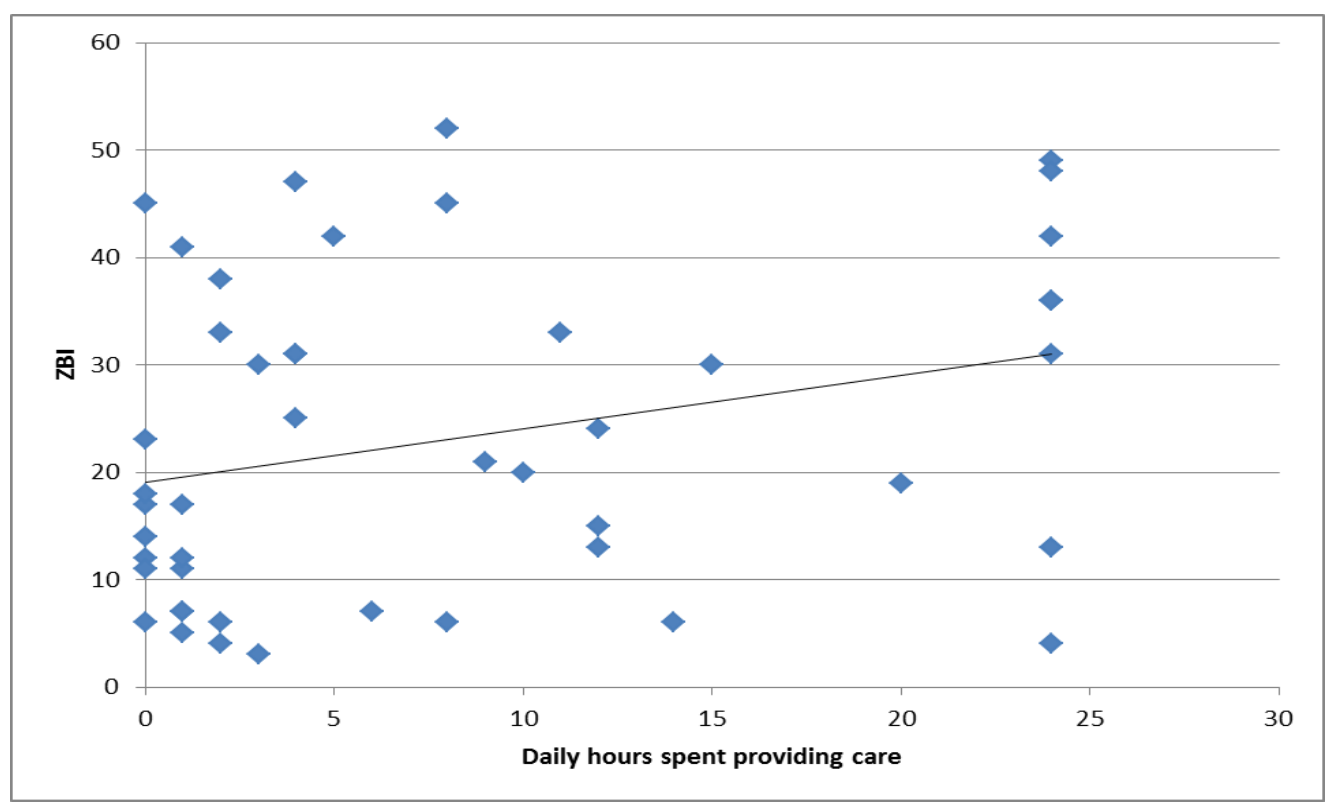

Figure 11. ZBI vs. Daily hours spent caregiving

With the amount of time committed to caregiving for an individual with ALS, working outside of the home is difficult. Yet, 36\% of the caregivers surveyed maintained employment, 
either part-time or full-time. However, continuing to work was not without consequence and these caregivers rated higher on overall burden with a p-value of 0.0399 (Figure 12). The stress of holding a job and working an average of eight hours caregiving in the home is substantial and it was reflected in the ZBI scores.

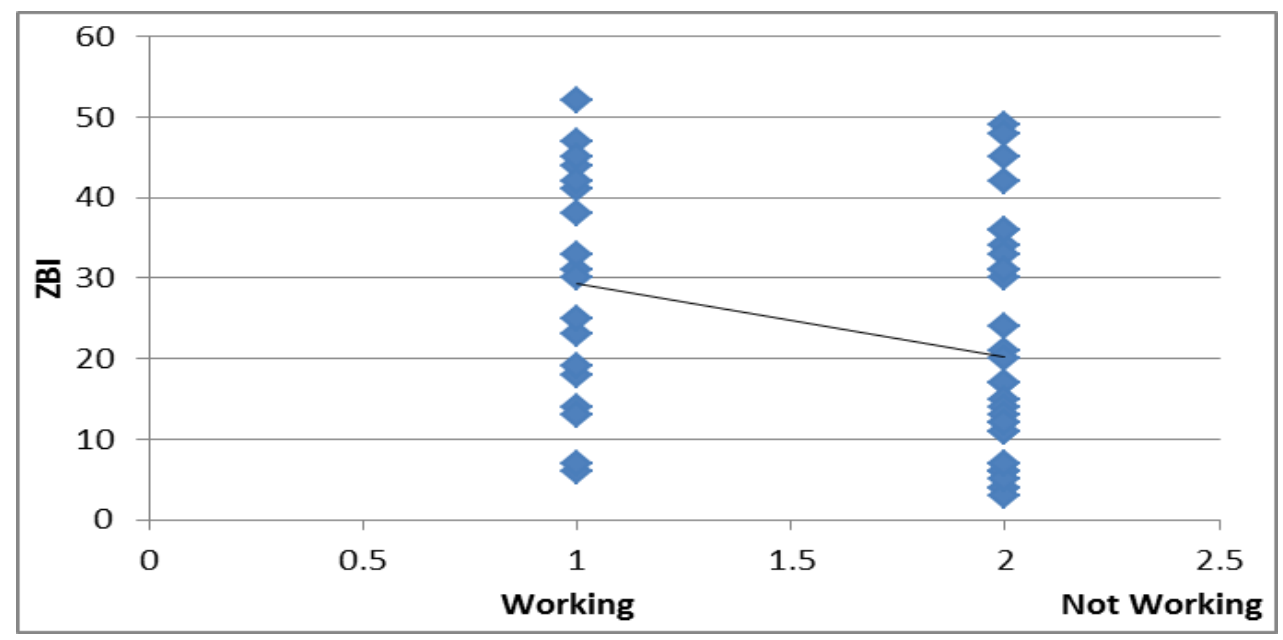

Figure 12. ZBI vs. Employment status

While the majority of this discussion has focused on factors that increase a caregiver's burden, it is important to recognize that one factor was found to reduce burden. Having an adequate support system correlated with lower burden ratings with a p-value of 0.0210 (Figure 13). Caregivers were not asked to describe their support system, but were simply asked whether the amount of social support they received was adequate or inadequate. Thirty-eight caregivers, $79 \%$ of those surveyed, rated their social support systems as adequate and had an overall lower burden average than the remaining individuals. While these social support systems most likely do not perform any caregiving duties or relieve caregiver responsibilities, having a friend or relative available for support is important. With the number of hours dedicated to patient care, many caregivers have less opportunity for social engagements. Feeling that they still have outside emotional support and assistance from other people in their community is important and contributory to lower burden levels. 


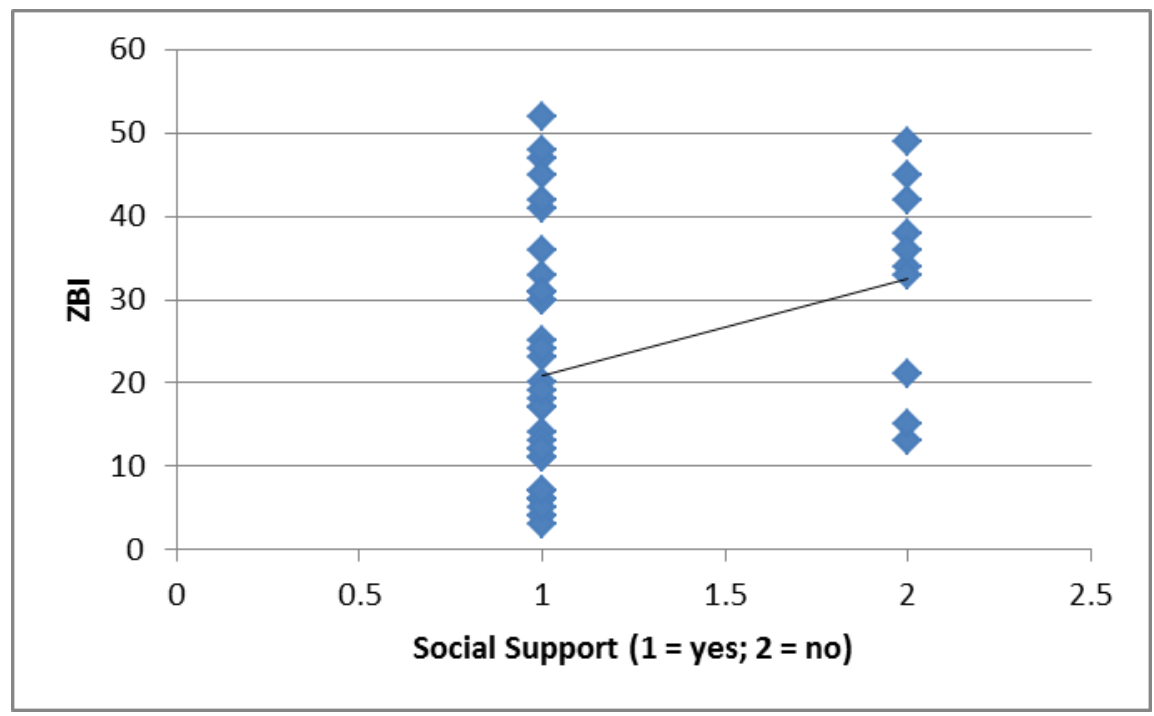

Figure 13. ZBI vs. Social support

Age, daily hours spent caregiving, and social support, and employment status were all statistically significant when compared with caregivers’ burden levels. Only age and the daily hours spent caregiving were statistically significant when compared with both the caregivers' burden levels and their depression symptoms. However, gender and religious affiliation were almost significant with p values of 0.0588 and 0.0531 respectively and are displayed in figures 14 and 15 below. Surveying these caregivers at different times or increasing the sample size may have altered statistical significance. As seen in figure 14, only three of the male caregivers ranged well above a ZBI score of 20, which indicates little to no burden. Conversely, similar to the women's BDI scores, the women caregivers scored much higher on the burden scale. Religious affiliation was less differentiated when compared with the ZBI. However, as noted in figure 15, none of the caregivers identifying as other Christian reported a score less than 20. Catholic and Protestant caregivers also reported high burden scores, but there were also Catholic and Protestant caregivers that reported little or no burden. If a larger sample of caregivers, either Catholic or Protestant, had noted lower burden scores, the p-value may have proven statistical significance. 


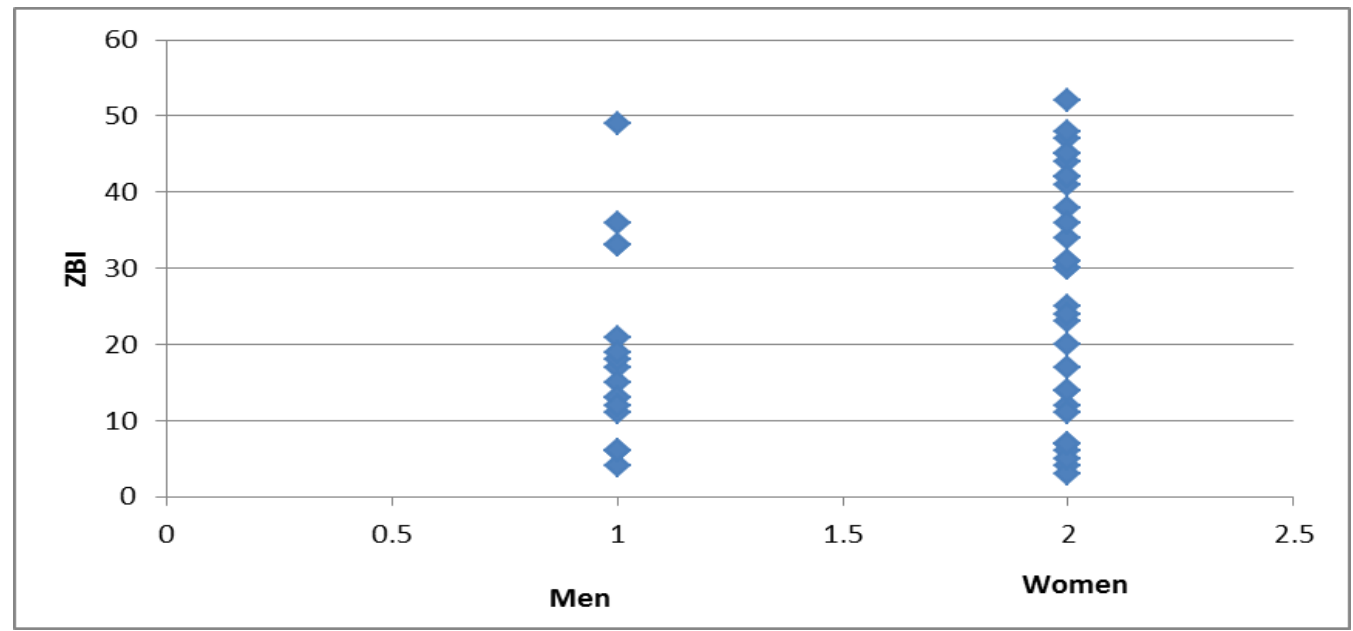

Figure 14. ZBI vs. Gender

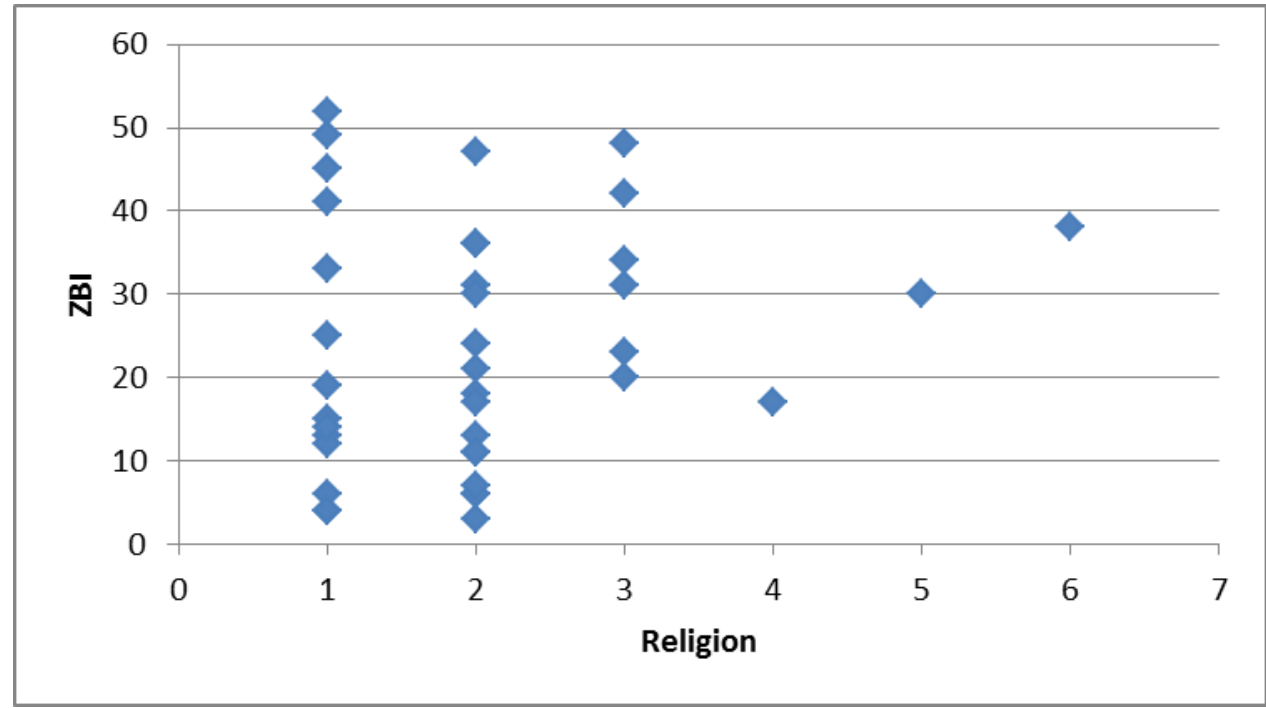

Figure 15. ZBI vs. Religious Affiliation

\subsubsection{Non-statistically significant other life factors}

No significant relationship was established between patient progression, weekly hours receiving caregiving assistance, living arrangement, household income, or education level when compared with depression symptoms or burden levels. Previous research had also found no relationship between education level and household income and caregiver depression (Rabkin, 2009). 
However, while these other factors did not have statistical significance in this study, they are worth examining further.

When looking at factors compared to the BDI, neither employment status, education level, and household income were statistically significant. This was somewhat surprising since these are all major aspects of the caregivers' lives. In terms of employment status, there was a large range of BDI scores for both our working and non-working caregivers resulting in a pvalue of 0.7072 (Figure 16). The difference in scores for working and non-working caregivers was that, with regards to BDI, many working caregivers had low BDI scores (Figure 12). Conversely, with ZBI, only a few working caregivers had low ZBI scores while most caregivers scored rather high on the ZBI scale if they still maintained employment. This emphasizes the importance of looking at caregiver burden and not simply focusing on depression as an evaluation of health.

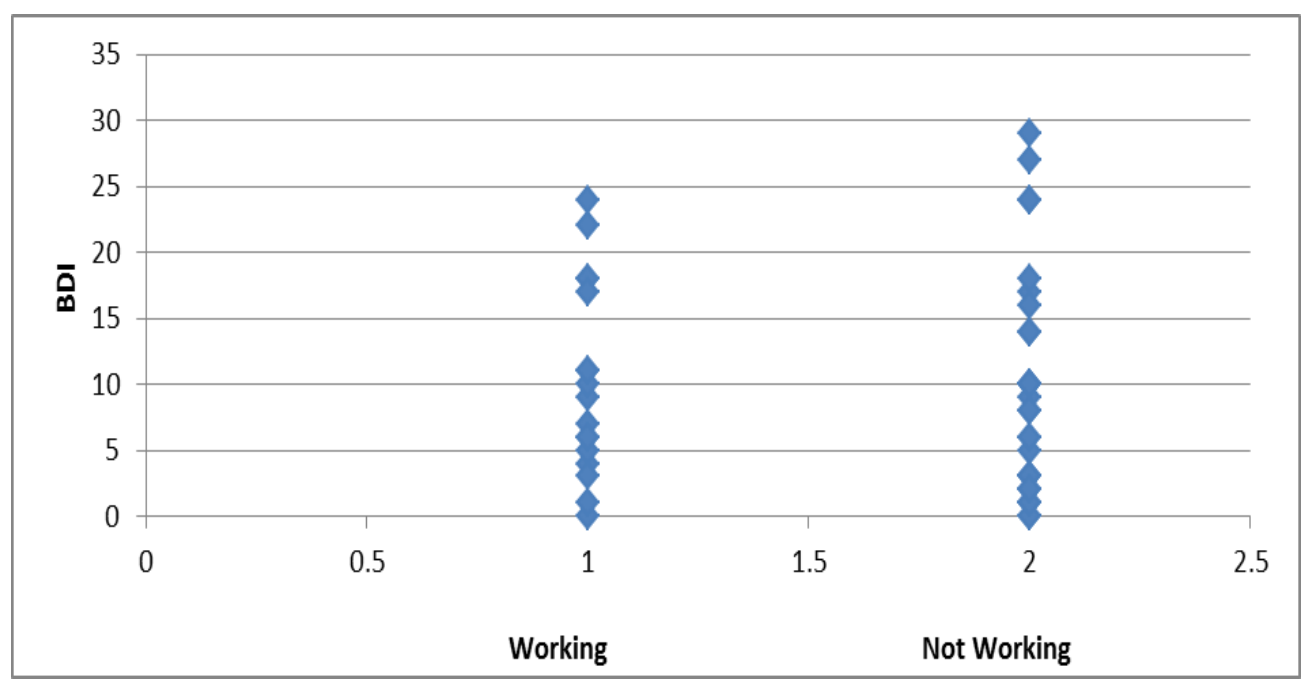

Figure 16. BDI vs. Employment status

Education level was not significant for either the BDI (Figure 17) or the ZBI (Figure 18). As seen in the figures below, BDI and ZBI scores were scattered across the board and p-values were 0.3263 and 0.9488 respectively. Of the 50 caregivers, 36\% had their high school diploma, 
$30 \%$ had attended some college, 30\% had completed college, and $2 \%$ had achieved a graduate degree. While only two caregivers noted that they had completed graduate level education, there was no clear pattern seen between only having attended high school and pursuing any further education. With respect to BDI, there appears to be a plethora of caregivers that had some college or completed college that reported lower BDI scores. However, when considering the BDI scores of the caregivers attending graduate school, there is not enough evidence to report a significant relationship.

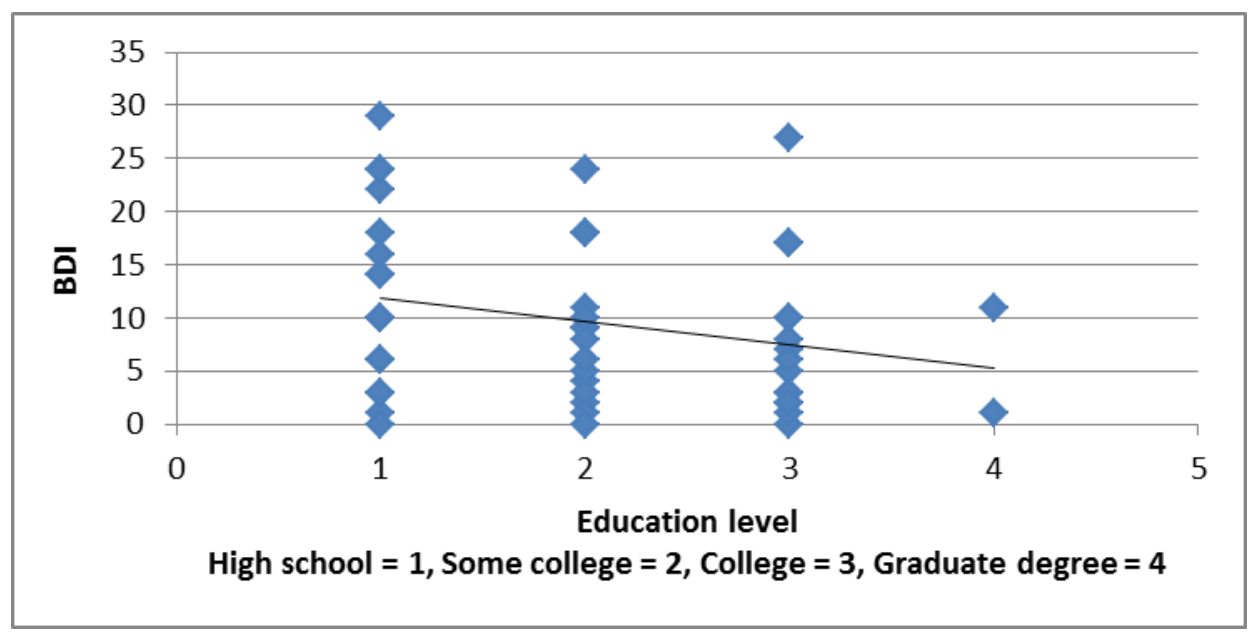

Figure 17. BDI vs. Education level

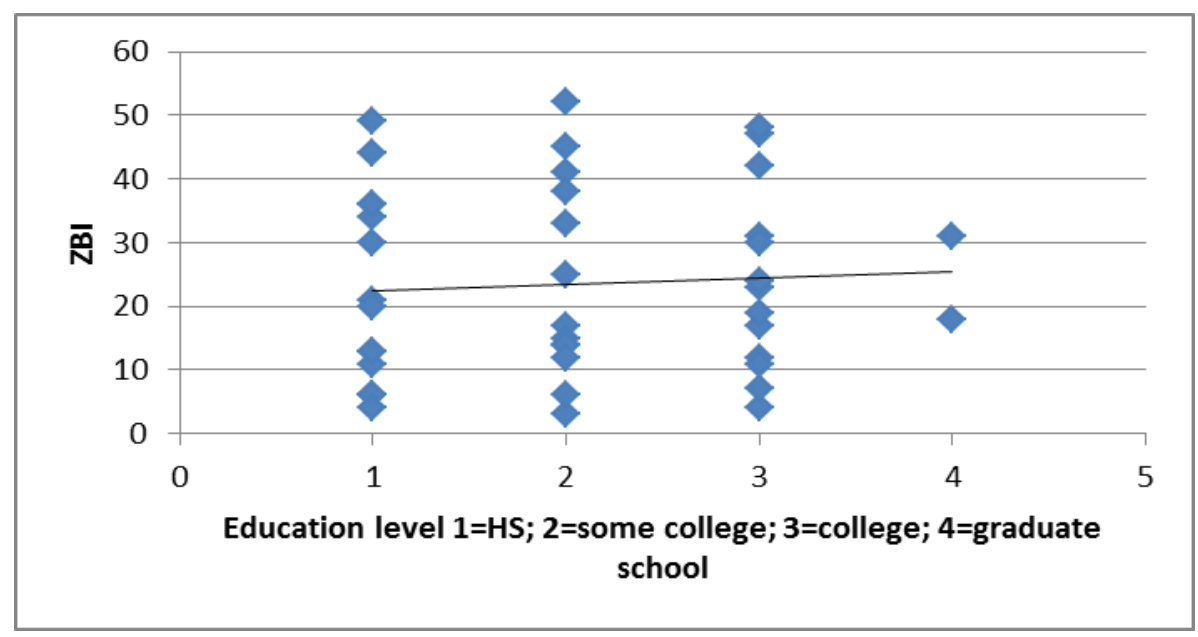

Figure 18. ZBI vs. Education level 
Household income was also very variable when compared with both BDI and ZBI, with p-values of 0.0931 and 0.3335 respectively. Since finance burden was significant with respect to BDI, one would expect household income to also be associated. This was not the case and there was no clear trend. It is important to note, however, that when asked about financial burden; whether there was a burden was up to the discretion of the caregiver. Caregivers were simply asked whether they felt their finances were significantly inadequate, somewhat inadequate, or adequate. Some caregivers budgeting with a household income of $\$ 40,000$ may feel their finances are adequate, whereas other caregivers making a household income of \$80,000 may report somewhat inadequate finances. The financial burden question was one of opinion and perception, while evaluating household income was purely quantitative. Recognizing this limitation, it is essential to also note that financial burden was not significant when compared to ZBI scores, with a p-value of 0.3082 (Figure 21). This is surprising since ZBI scores are also based on the opinion and feelings of the caregiver. It is difficult to determine why BDI scores would be higher with a greater financial burden but ZBI scores remain dispersed.

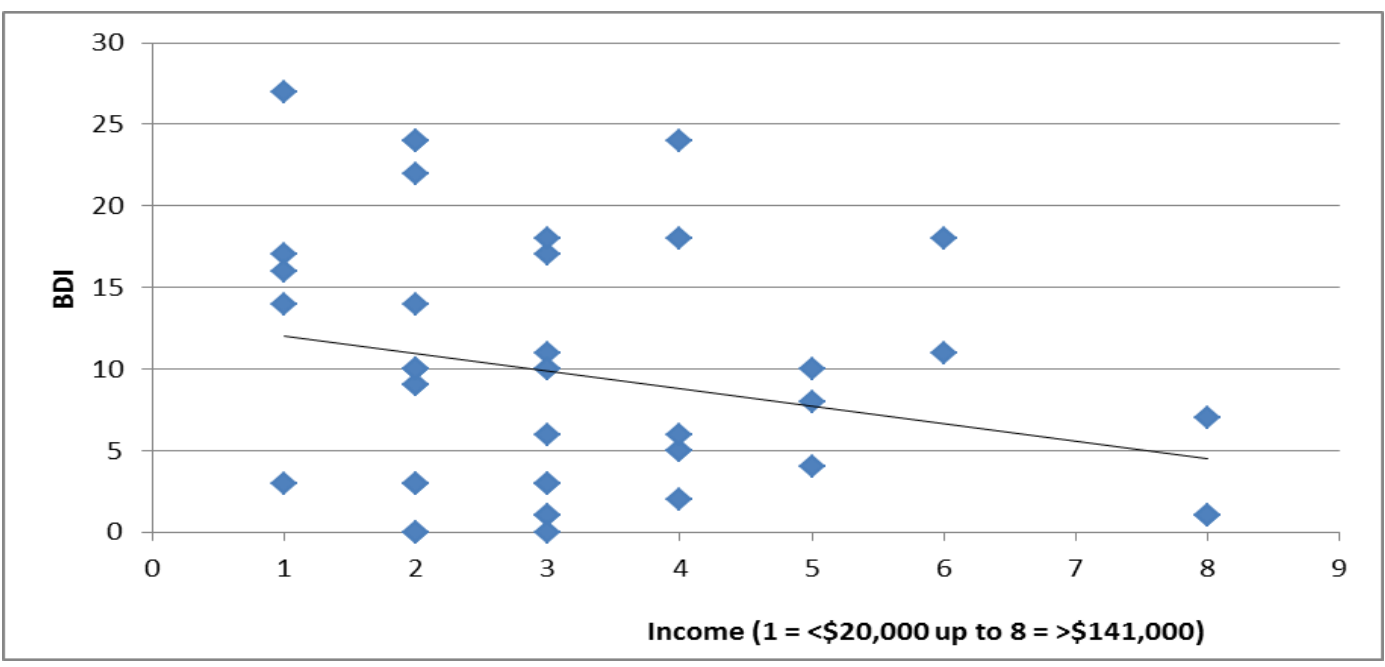

Figure 19. BDI vs. Household income 


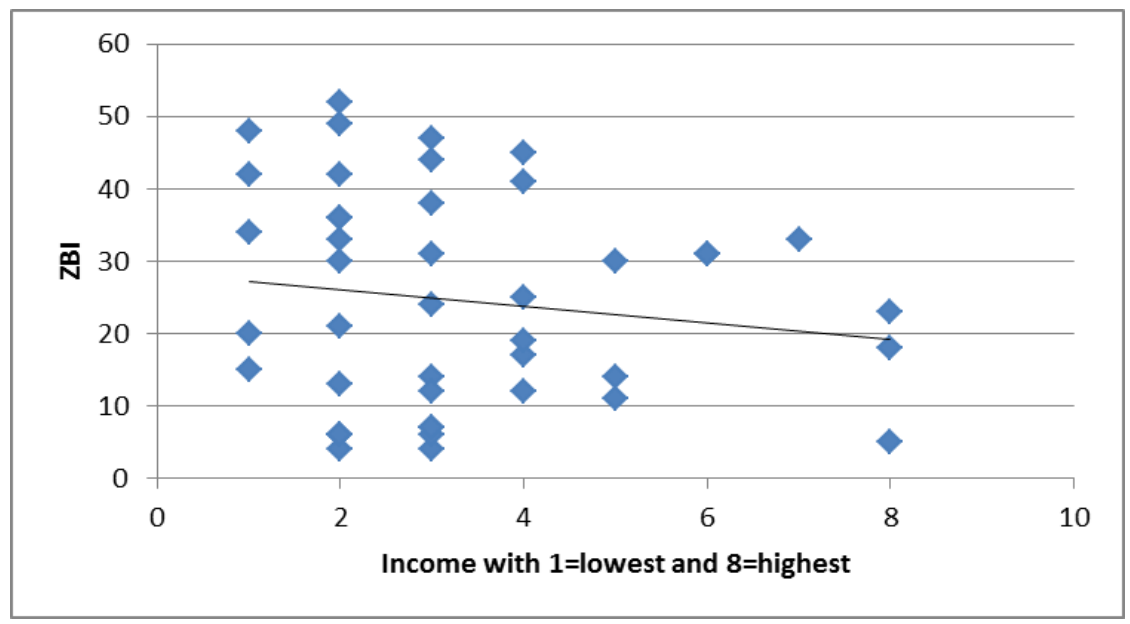

Figure 20. ZBI vs. Household income

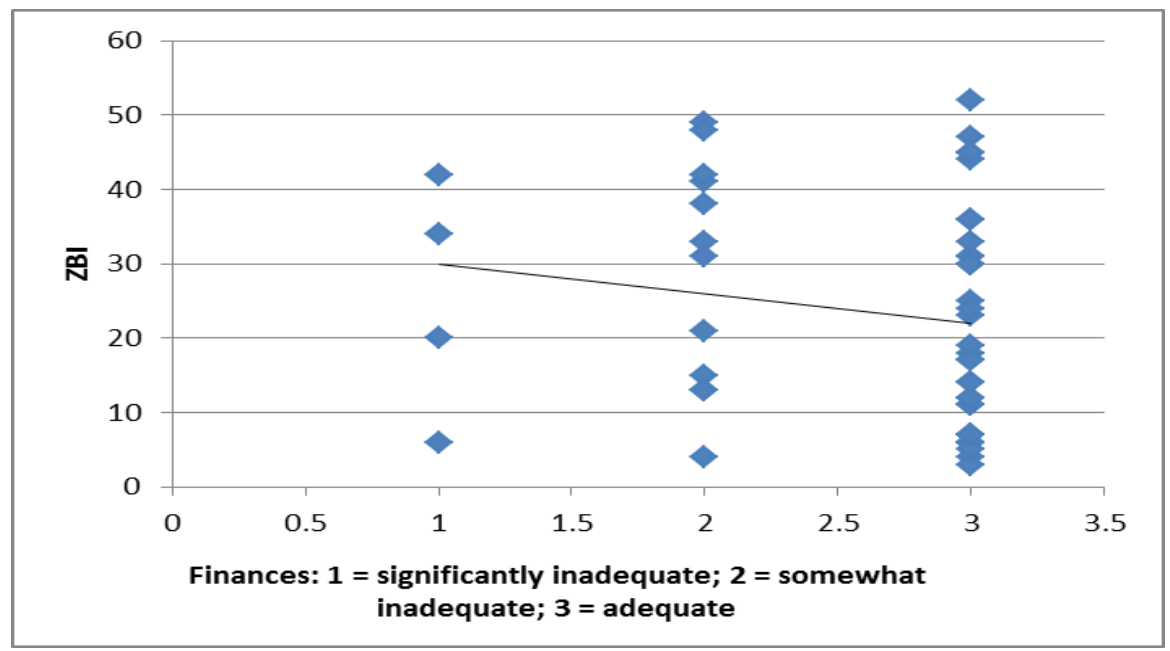

Figure 21. ZBI vs. Financial burden

Another factor in the caregivers' lives that we studied was their living arrangements. Were the caregivers living only with a spouse, were there still children in the home, or was there a different household composition? This data could have been skewed either way. Living with just an affected individual could be straining since there is no one else to assist with providing care. On the other hand, having other people in the house may cause additional pressure since there may be more responsibilities either as a parent or other relative. Nevertheless, in evaluating the caregivers, there was no association between the living arrangement of the caregiver and their depression or burden (Figure 22, Figure 23). Both BDI and ZBI scores were inconsistent 
and had calculated p-vales of 0.8288 and 0.3470 respectively. Remarkably, there was only one caregiver that reported living with family besides a spouse or children and only one caregiver that reported living alone. The caregiver that lived alone cared for a friend but still chose to live in a separate residence. These two caregivers were not included in the statistical analysis. So with a limited sample size, the comparison was between caregivers living with a spouse, $68 \%$ of study participants, and caregivers living with a spouse and children, 28\%. In future research, it would be interesting to ask the ages of the caregivers' children. Maybe caregivers living with their spouse and older children register lower depression and/or burden than caregivers living with their spouse and young children. Having that additional information may differentiate better between the caregivers' living arrangements and their BDI and ZBI scores.

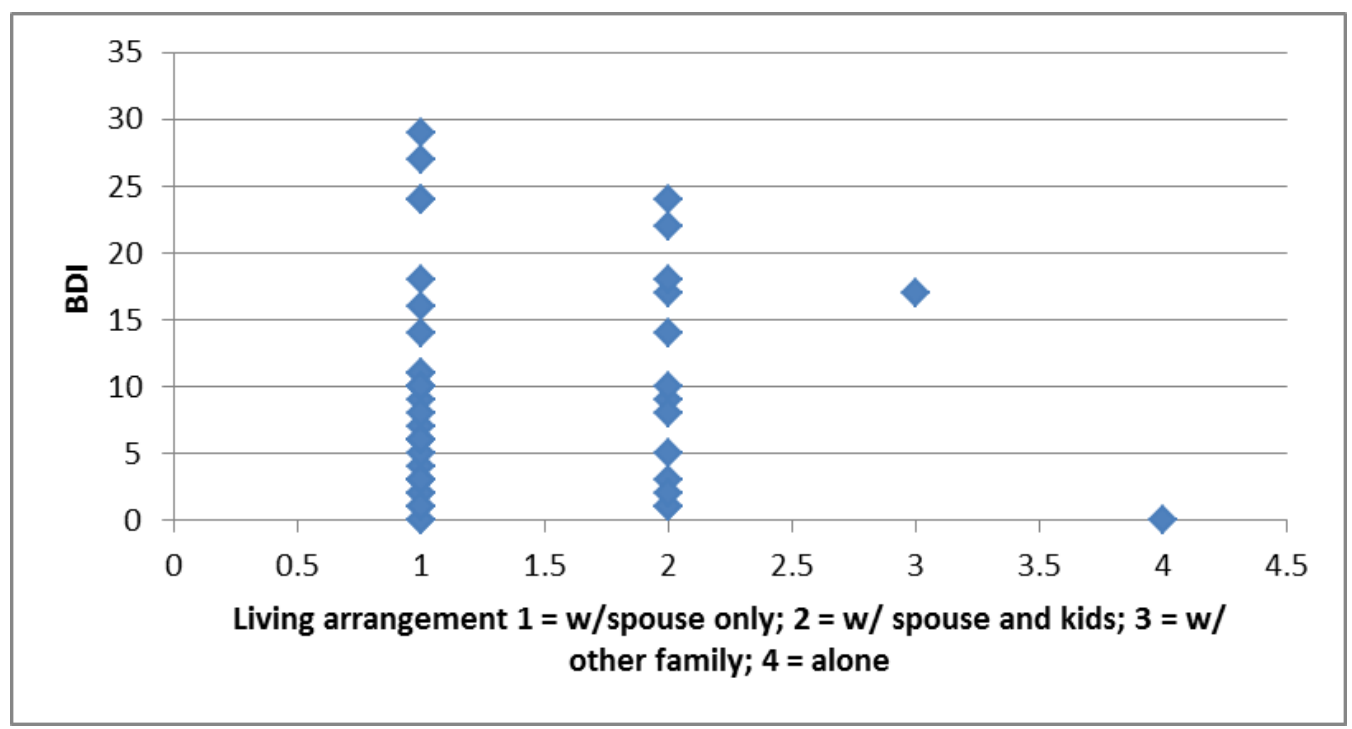

Figure 22. BDI vs. Living arrangement 


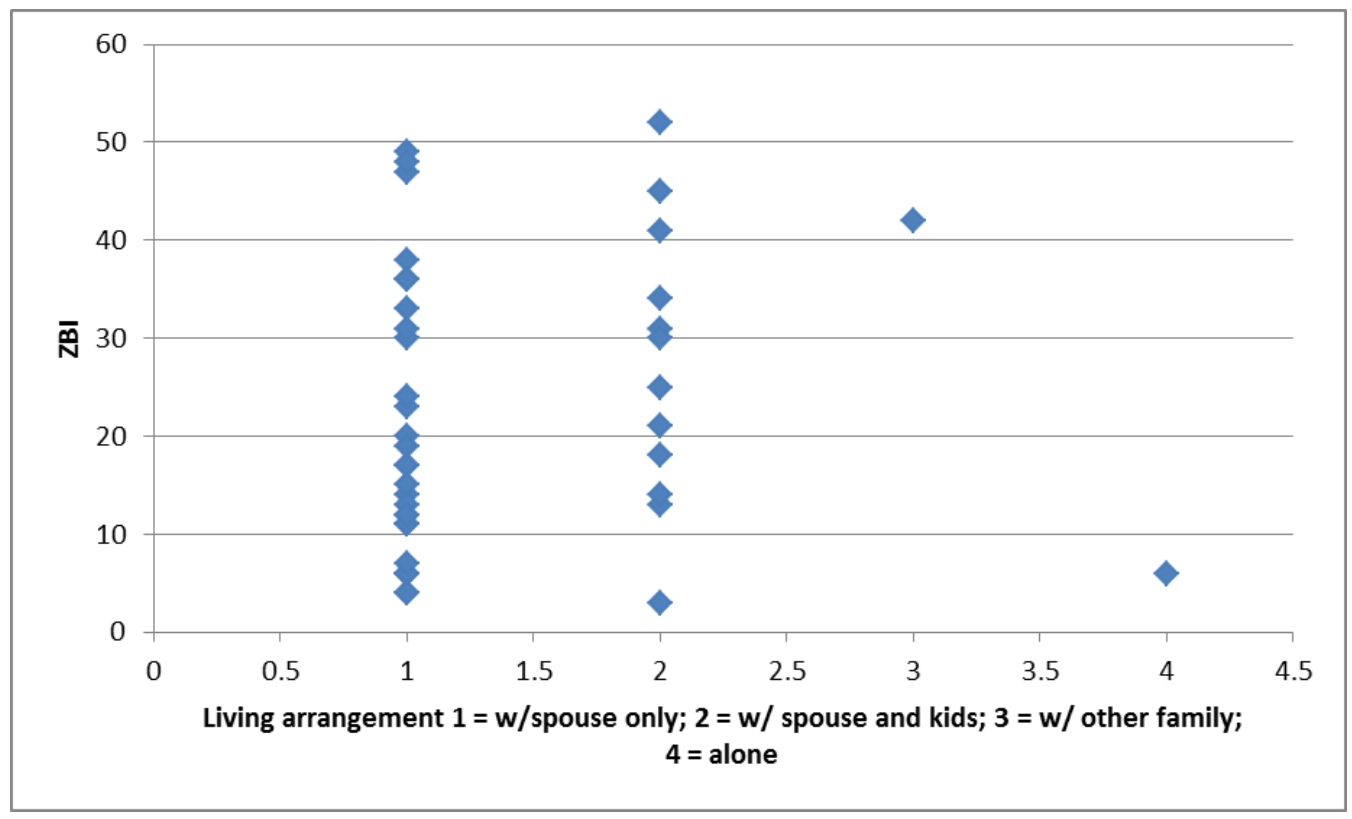

Figure 23. ZBI vs. Living arrangement

Living arrangement was an important aspect to consider as it provided insight into the caregivers' lives, asking whether there were other people to help out within the home. This study also wanted to assess whether having outside help, either in the form of a paid caregiver, volunteer, or friend, contributed to or lessened caregiver depression and burden. Caregivers were asked whether they received any outside help and 37\% reported they did and 63\% claimed they did not. Yet, in assessing those responses versus the BDI and ZBI scores, there was no clear relationship, with p-values of 0.4230 and 0.4061 . The number of hours of help received per week was also not significant, with p-values of 0.9471 when compared with BDI and 0.4098 when compared with ZBI. As seen in figure 24 and figure 25 below, the majority of our caregivers recorded 0 hours of help/no help, leaving the remaining caregivers varying widely. Considering that the daily hours caregiving for the patient was significant when compared to BDI and nearly significant when compared to ZBI, it is astounding that there is no correlation with the number of hours of outside help received. One would think that having more outside help would equate with lower burden scores. Again, this may be difficult to establish without probing further. It is 
possible that caregivers receiving more outside help may be caring for sicker individuals. So while the number of hours they have help is greater, the strain and anxiety they are experiencing is still high. The outside help provided could also be varied among households. The question was left up to the interpretation of the caregiver and the data is dependent on how many hours per week the caregiver felt he/she received outside assistance. These nuances are interesting and could be very different in the future depending on the attitudes, opinions, and emotions of the study population.

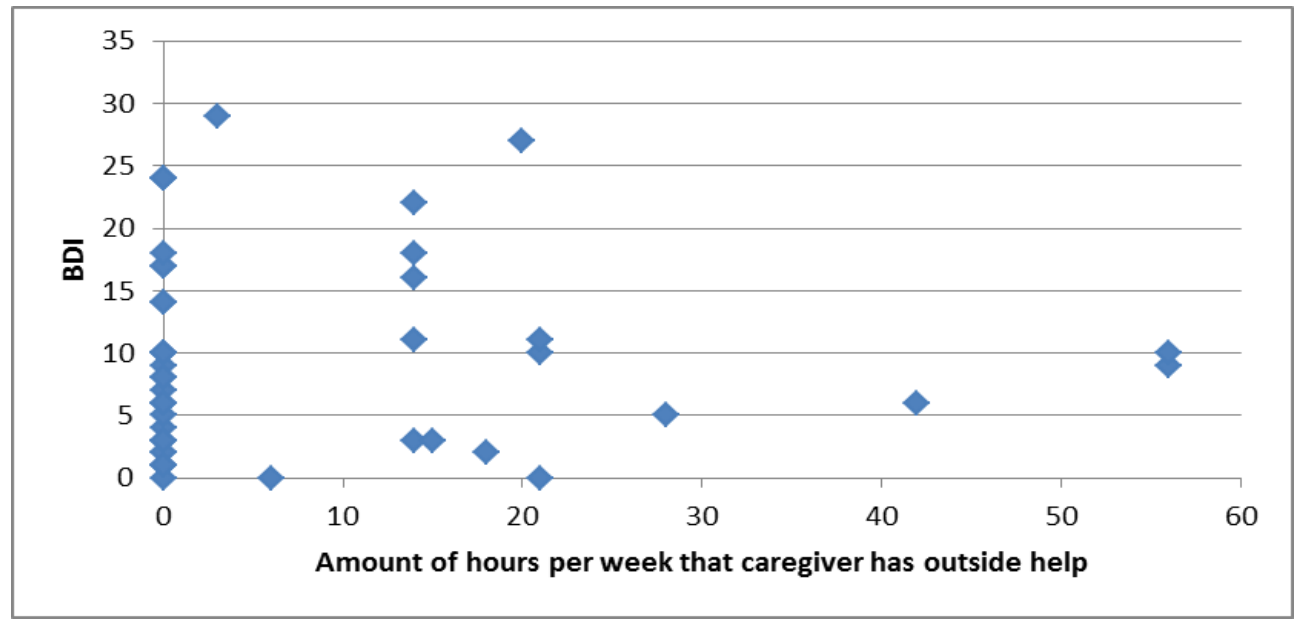

Figure 24. BDI vs. Hours of outside help received per week

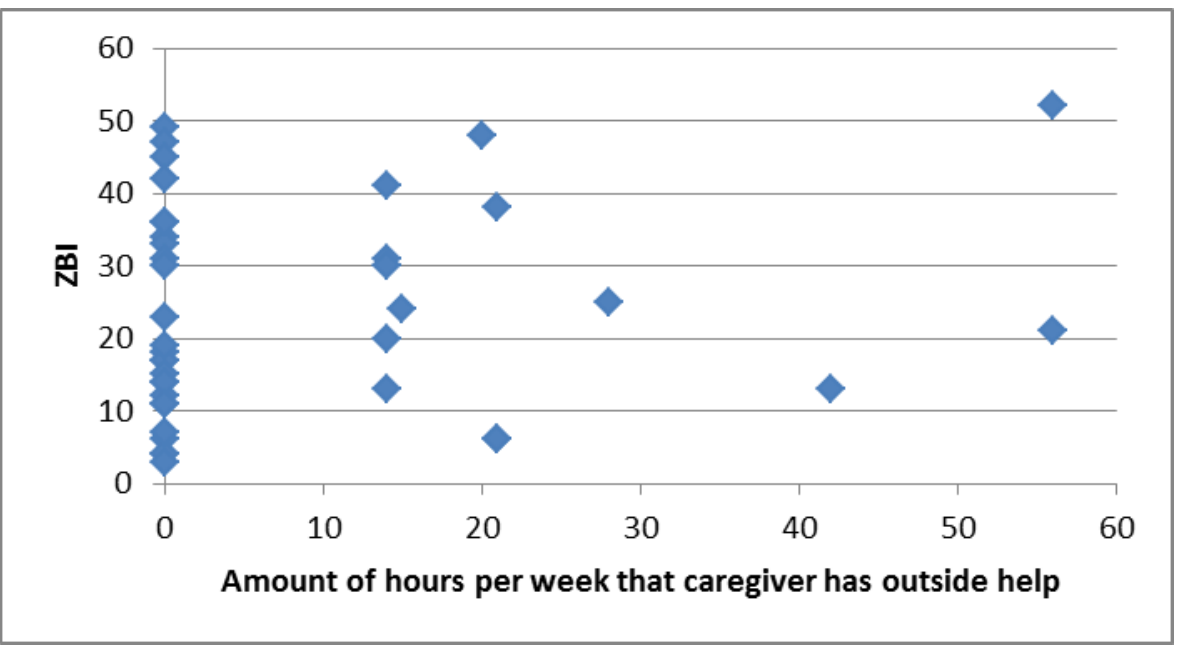

Figure 25. ZBI vs. Hours of outside help received per week 
While we have already discussed the association between religious denomination and BDI score, as well as the probable relationship between religious denomination and ZBI score, but it is also worth addressing the additional religion questions that were asked. Caregivers were asked whether they considered religion as a part of their daily life and how religious they considered themselves. Fifty-four percent of caregivers believed that religion did play a part in their daily lives, while the other $46 \%$ of caregivers disagreed. There was no correlation between religion as a part of daily life when compared with either the BDI or ZBI score, with p-values of 0.6185 and 0.3307 respectively (Figure 26, Figure 27). Likewise, no relationship was found between BDI or ZBI when compared with how the caregivers rated their religiousness. Caregivers were able to choose whether they considered themselves very religious (21\%), religious (37\%), a little religious (17\%), or not religious (25\%). Caregiver responses were varied and no statistical significance was determined, with p-values of 0.9750 for BDI and 0.6758 for ZBI. These results, differing from Calvo et al. (2011), make one question the significance of religious affiliation. It is possible that the correlation found between BDI and religious denomination was merely a result of having a strong social support network through the church and not the result of practicing a specific religion.

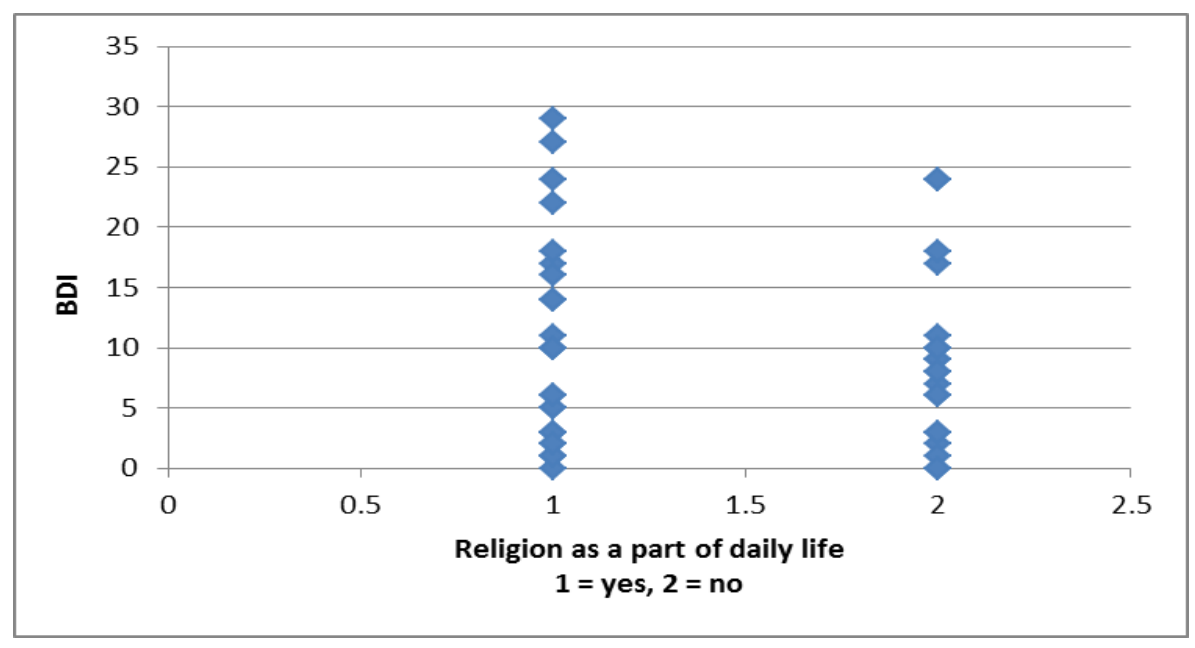

Figure 26. BDI vs. Religion as a part of daily life 


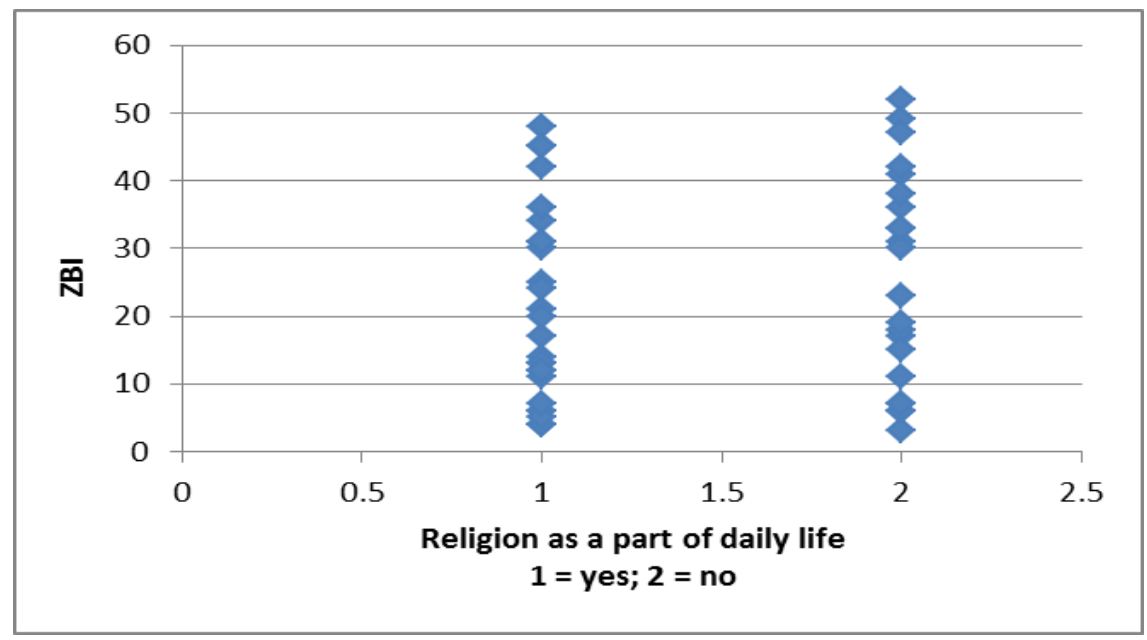

Figure 27. ZBI vs. Religion as a part of daily life

Investigating further into the intricacies of the religion data leads to the last correlate we examined. As mentioned previously, social support was the only factor found to be protective and caregivers with adequate social support had lower burden scores overall (Figure 13). Interestingly, there was no statistical significance when social support was compared with BDI scores, with a p-value of 0.1100 . On examining the graph below, however, can notice that only one caregiver without an adequate social support system had a BDI score less than 10. Perhaps without this outlier, the p-value would have been different, since a large number of caregivers with adequate social support had low BDI scores.

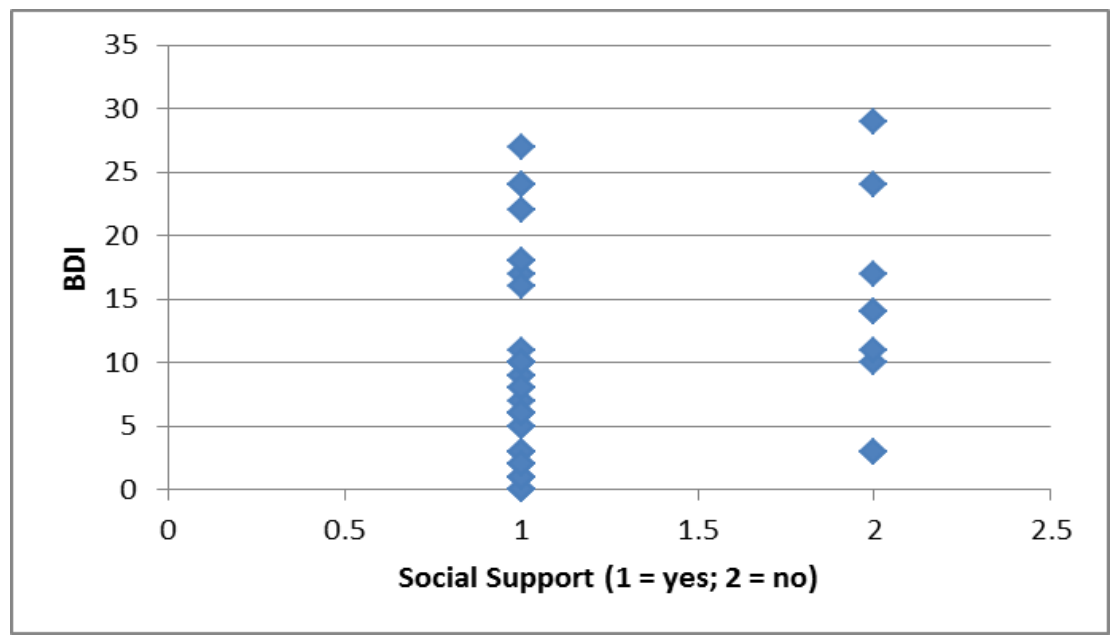

Figure 28. BDI vs. Social support 


\subsection{DISCUSSION}

\subsubsection{Implications for ALS caregivers}

The importance of this study was to establish a factor or factors in a caregiver's life that are contributory to increased caregiver depression and/or increased caregiver burden. Multiple depression symptoms and/or burden equate with an overall decrease in quality of life. Determining which factors in their lives negatively impact ALS caregivers would allow health professionals to better anticipate and recognize depression symptoms. Even more importantly, identifying stressors allows for the implementation of intervention services. Preventative measures and the establishment of caregiver assistance would hopefully help reduce caregiver burden and lead to an overall better quality of life while caregiving.

While some contributory depression and burden factors, such as age and gender, cannot be changed, this is still vital information. Knowing that younger caregivers and female caregivers have a higher risk for depression and may feel more overall burden allows one to counsel these caregivers differently and offer more appropriate services. Religion is another factor that is non-adjustable. Similarly, however, knowing someone’s religious denomination can predict their likelihood for depression symptoms or burden. If an individual does not identify with a religion, their risk for depression may be substantially greater. 
Other aspects of caregivers' lives that cause depression and/or burden are most malleable. Support programs and assistance can be provided to help with financial burdens, hours spent caregiving, and social support. Although it is unrealistic to provide financial assistance to every ALS patient-caregiver pair, caregivers requiring financial support can be guided to Social Security programs or other government assistance agencies. Social workers can work with these caregivers early on so financial assistance is in place before caregivers experience monetary strain. Likewise, while caregivers will always have some time commitment with caregiving, paid caregivers or outside help can be provided. Insurances will only cover so many hours of caregiving assistance per week, month, or year. Therefore, it is essential that health professionals working with ALS patients recognize this limitation. Social workers or other professionals can assist with obtaining paid help if that is a possibility. For caregivers that cannot afford paid help, other options could be explored. Volunteer programs could be established. These programs could be as complex as providing free in home care by qualified caregivers or as simple as delivering meals, doing laundry, or providing cleaning services to relieve other caregiver responsibilities. This support would extend past the medical needs of the patient and focus on caregiver support and family care.

Lastly, and probably the easiest factor to manipulate, is the aspect of social support. For caregivers that do not feel that they have an adequate social support system, support groups and social outings should be options. The Muscular Dystrophy Association (MDA) currently runs a support group in Pittsburgh that welcomes ALS families and there is an online support group option in Johnstown, Pennsylvania. However, having an additional option may prove beneficial. Offering a support group exclusively for caregivers will provide a forum in which they can feel comfortable sharing in a safe, un-stressful environment. It is imperative to also keep in mind that 
offering a support group may not be enough to completely relieve caregiver burden. Babysitting and transportation will need to be considered. Having a support group will be unhelpful if caregivers are unable to get there or cannot find child care. These are all facets that will need to be considered, but it is promising to know that caregiver depression and burden may be reduced if further steps are taken.

Even though there are a number of details that will need to be taken into consideration, establishing a support group specific to ALS caregivers is the first essential step in helping to relieve depression and burden. It could be scheduled in tandem with the regular support group so that transportation could be coordinated. However, this time away from the patient is essential and will allow the caregivers to talk freely without inhibiting or disguising their true emotions. Since we have determined that social support can be a protective factor with regards to BDI, ensuring that social support groups are an option is imperative. Other interventions can and should be put in place, but these can be organized less immediately.

\subsubsection{Public Health Significance}

Depression is a significant problem in the United States, a problem that continues to grow. According to data from the Center for Disease Control, 9.1\% of Americans suffer from depression (CDC, 2010). Decreasing the risk for depression, even for a smaller population, is substantial. Helping reduce depression symptoms and relieving burden for ALS caregivers is of public health significance. Interventions for ALS caregivers, if shown to be effective, would serve as a template for caregivers of other degenerative neurological conditions. Many conditions are similar in their progressive nature and fatal end result. Finding intervention strategies for ALS caregivers and protective approaches against increasing depression and 
feelings of burden or anxiety can translate to a plethora of other disorders. With 52 million caregivers in the United States, any research evaluating ways to preserve caregiver quality of life is critical.

\subsubsection{Future research}

The data provided in this paper is informative and has considerable implications for ALS caregivers and general public health. Future research, however, would be beneficial to elaborate on the information obtained. This was a cross-sectional study, evaluating caregivers' perception of burden and depression symptoms at that specific period in time. It would be valuable to perform a longitudinal study, comparing caregivers' answers over a period of time. It would be interesting to see how answers compared between individuals who have provided care for a short period of time versus caregivers who have provided care for multiple years. It would also be useful to determine at which point during caregivers' experiences social support should be offered and discussed. Additional factors in the caregivers’ lives could be evaluated as well.

\subsubsection{Limitations}

There were some limitations associated with this study. The main limitation was the sample size. While recruitment was excellent, the available ALS population is smaller than with other conditions. Obtaining a larger sample size was also difficult as not all caregivers were eligible for this study. Future research may consider conducting a multi-center study in order to increase participant numbers and further diversify the study population. Additionally, only the Beck 
Depression Inventory and Zarit Burden Interview were used as the tools to identify depression and burden. Other assessments may provide additional clarity or information. Other studies could use quality of life measurement tools as well for a different perspective in evaluating caregivers. 


\subsection{CONCLUSION}

Caregiver burden and acquirement of depression symptoms are a significant problem, especially among caregivers for fatal and progressive neurodegenerative conditions like Amyotrophic Lateral Sclerosis. Until this study, no one had explored how different factors in an ALS caregiver's life affected his/her risk for developing depression and his/her sense of daily burden. This research sought to provide specific information regarding which aspects of caregivers' lives produced the most stress and anxiety with the hope that interventions and preventative services could be implemented to maintain caregiver health and happiness. Factors were found to be risk factors for depression symptoms and increased burden. This provides insight on to how to best approach the problem of caregiver burden and preserve their quality of life. Further research should be conducted to pursue this avenue, as better understanding risk factors can facilitate caregiver support. 


\section{CONSENT FORMS}

\section{CONSENT TO ACT AS A PARTICIPANT IN A RESEARCH STUDY}

TITLE: Perception of Life Changes in ALS Caregivers

PRINCIPAL INVESTIGATOR:

Kristen Qutub

Genetic Counseling Intern

University of Pittsburgh

Graduate School of Public Health

Telephone: 908-839-8794

CO-INVESTIGATORS: David Lacomis, M.D.

Professor of Neurology and Pathology

University of Pittsburgh

UPMC Presbyterian

Telephone: 412-647-1706

Robert Ferrante, Ph.D., MS

Professor of Neurological Surgery

University of Pittsburgh

Scaife Hall 
Telephone: 412-802-6497

\author{
Stephen Albert, Ph.D. \\ Professor of Behavioral and Community Health Sciences \\ University of Pittsburgh \\ Graduate School of Public Health
}

Telephone: 412-383-8693

Eleanor Feingold, Ph.D.

Professor of Human Genetics

University of Pittsburgh

Graduate School of Public Health

Telephone: 412-383-8599

Sasa Zivkovic, M.D.

Associate Professor of Neurology

University of Pittsburgh

UPMC Presbyterian

Telephone: 412-692-4917

\title{
Why is this research being done?
}

You are being asked to participate in a research study in which we will look at multiple factors within a caregiver's life and whether or not these specific factors correlate with caregivers' quality of life and/or depression symptoms. Having a better understanding of specific stressors within caregivers' lives will help to develop future specialized intervention programs and support systems. 


\section{Who is being asked to take part in this research study?}

Primary caregivers of ALS patients are eligible for this study.

Additional criteria include the following:

1. Caregivers must spend a minimum of 3 hours daily attending to needs of the patient. This includes, but is not limited to, basic life tasks, transportation, and emotional support.

2. For this study, paid help (either live-in or within the home) will not be included. Staff within nursing homes, hospice centers, and group homes will not be included in this caregiver study. Caregiver volunteers will be included, as long as they care for the patient a minimum of 3 hours per day.

3. If a patient has multiple caregivers, only the primary caregiver (caregiver who spends the greatest amount of time taking care of the patient) will be included in the study.

4. Caregivers must be over the age of 18 .

\section{What procedures will be performed for research purposes?}

If you decide to take part in this research study, you will undergo the following procedures:

\section{Experimental Procedures:}

If qualified to take part in this research study, participants will be asked to complete surveys. The surveys will ask participants about basic demographic information, questions about their daily life, and questions regarding their emotional health and feelings. The surveys should take approximately 15-20 minutes to complete.

The patient will be asked for his/her consent to obtain information from the patient's medical or clinic records. This information will be used in analysis with data collected from the caregiver. If the patient does not consent to participating in this study, caregivers will not be able to participate and will not be enrolled in this research study. 


\section{Additional Procedures:}

Participants will be asked to complete the above surveys a total of three times, at sessions three months apart. These additional commitments will take place at future clinic visits. If we are unable to personally meet in clinic three times, a phone interview will be conducted in order to administer the surveys.

\section{What are the possible risks, side effects, and discomforts of this research study?}

The possible risks of this research study may be increased emotional stress of discomfort. Some of the questions ask about at home living situations, personal feelings, or financial status. Some of these questions may be uncomfortable or difficult to answer. In answering some of the questions, one may experience emotional stress, including increased sadness, discomfort, or grief. A breach of confidentiality is also a potential risk.

\section{What are possible benefits from taking part in this study?}

You will likely receive no direct benefit from taking part in this research study. Should the study find specific aspects of caregivers' lives that contribute to a decrease in quality of life, however, additional support systems and intervention programs can be created in the future. These new programs and family support options will be beneficial to caregivers in the future.

If I agree to take part in this research study, will I be told of any new risks that may be found during the course of the study?

You will be promptly notified if, during the conduct of this research study, any new information develops which may cause you to change your mind about continuing to participate. 


\section{Will there be any associated costs with this study?}

There will be no costs to participants within this study.

\section{Will I be paid if I take part in this research study?}

There is no monetary compensation for study participants.

\section{Who will know about my participation in this research study?}

Any information about you obtained from this research will be kept as confidential (private) as possible. Only investigators associated with this study and their research staff will have access to collected identifiable data. All records related to your involvement in this research study will be kept private. Your identity on these records will be indicated by a case number rather than by your name, and the information linking these case numbers with your identity will be kept separate from the research records.

At the end of this study, any records that personally identify you will remain stored in locked files and

will be kept for a minimum of seven years. Your identity will not be revealed in any description or publications of this research. Data may be shared with others, but it will be shared without identifiers.

In unusual cases, your research records may be released in response to an order from a court of law. It is also possible that authorized representatives from the University of Pittsburgh Research Conduct and Compliance Office may review your data for the purpose of monitoring the conduct of this study. Also, if the investigators learn that you or someone with whom you are involved is in serious danger or potential harm, they will need to inform the appropriate agencies, as required by Pennsylvania law. 


\section{Is my participation in this study voluntary?}

Yes! Your participation in this study is completely voluntary. You may refuse to take part in it, or you may stop participating (withdraw) at any time, even after signing this form.

Your decision will not affect your relationship (or your loved one's relationship) with the University of Pittsburgh, the University of Pittsburgh Medical Center, or a health care insurance provider; nor will you (or your loved one) lose any benefits that you might be eligible for because of what you decide.

Any information collected prior to the date that you withdraw your consent may continue to be used by the investigators for the purposes described above. You may be withdrawn from the study at any time by the investigators: for example, if you were found to meet any of the study criteria that would exclude you from participating.

\section{VOLUNTARY CONSENT}

The above information has been explained to me and all of my current questions have been answered. I understand that I am encouraged to ask questions about any aspect of this research study during the course of this study, and that such future questions will be answered by the primary investigator listed on the first page of this consent document at the telephone number given.

I understand that I may contact the Human Subjects Protection Advocate of the IRB Office, University of Pittsburgh (1-866-212-2668) to discuss problems, concerns, and questions; obtain information; offer input; or discuss situations that have occurred during my participation.

By signing this form, I agree to participate in this research study. A copy of this consent form will be given to me. 
Printed Name of Participant

\section{CERTIFICATION of INFORMED CONSENT}

I certify that I have explained the nature and purpose of this research study to the above-named individual(s), and I have discussed the potential benefits and possible risks of study participation. Any questions the individual(s) have about this study have been answered, and we will always be available to address future questions as they arise.”

Printed Name of Person Obtaining Consent

Signature of Person Obtaining Consent
Role in Research Study

Date 


\section{AUTHORIZATION FOR THE RELEASE OF MEDICAL RECORDS FOR PARTICIPATION IN A RESEARCH STUDY}

TITLE: Perception of Life Changes in ALS Caregivers

$\begin{array}{ll}\text { PRINCIPAL INVESTIGATOR: } & \begin{array}{l}\text { Kristen Qutub } \\ \text { Genetic Counseling Intern }\end{array} \\ & \text { University of Pittsburgh } \\ & \text { Graduate School of Public Health } \\ & \text { Telephone: 908-839-8794 } \\ \text { CO-INVESTIGATORS: } & \text { David Lacomis, M.D. } \\ & \text { Professor of Neurology and Pathology } \\ & \text { University of Pittsburgh } \\ & \text { UPMC Presbyterian } \\ & \text { Telephone: 412-647-1706 } \\ & \text { Robert Ferrante, Ph.D., MS } \\ & \text { Professor of Neurological Surgery } \\ & \text { University of Pittsburgh } \\ & \end{array}$

Telephone: 412-802-6497

Stephen Albert, Ph.D.

Professor of Behavioral and Community Health Sciences

University of Pittsburgh 
Graduate School of Public Health

Telephone: 412-383-8693

Eleanor Feingold, Ph.D.

Professor of Human Genetics

University of Pittsburgh

Graduate School of Public Health

Telephone: 412-383-8599

Sasa Zivkovic, M.D.

Associate Professor of Neurology

University of Pittsburgh

UPMC Presbyterian

Telephone: 412-692-4917

\section{Why is this research being done?}

You are being invited to participate in this research study because your primary caregiver has agreed to participate. For this research study, researchers are assessing multiple factors in the lives of ALS caregivers. One of these factors is the current health status of the individual he/she is caring for. In order to best assess patient health, this study will look at patient ALS-FRS scores as well as clinic notes that are most relevant to your condition and function, including breathing, eating, mood, thinking, and mobility.

Who is being asked to take part in this research study? 
Primary caregivers of ALS patients are eligible for this study, if they meet the designated caregiver criteria of the study. Individuals cared for by the primary caregivers will also be asked to participate by providing access to their ALS-FRS score and clinic notes.

\section{What information will be disclosed?}

We will obtain information concerning your mobility, nutrition, breathing, and medications. Your ALS-FRS patient score will be collected. In addition, information used will include all current and past medications, cognitive problems or depression, any assistive devices used currently or in the past, whether a feeding tube has been placed, and your forced vital capacity.

\section{Why is this information needed?}

This information is necessary to evaluate if patient progression affects caregiver quality of life and how it compares to the effects other areas of the caregivers' lives have in regard to quality of life.

\section{Who is requesting this information and how long is this Authorization valid?}

The investigators listed above are requesting your authorization to use the medical records listed above. This Authorization will be valid for an indefinite period of time.

\section{What are the possible risks, side effects, and discomforts of this research study?}

There is a potential risk of breach of confidentiality. Although we will do everything in our power to protect your privacy and the confidentiality of your records, we cannot guarantee the privacy of your research records, including information that we obtained from your medical records. Only researchers listed above and 
their staff will have access to any identifying information. All data analysis will be done without personal identifiers.

\section{What are possible benefits from taking part in this study?}

You will likely receive no direct benefit from taking part in this research study. Should the study find specific aspects of caregivers' lives that contribute to a decrease in quality of life, however, additional support systems and intervention programs can be created in the future. These new programs and family support options will be beneficial to caregivers in the future.

If I agree to take part in this research study, will I be told of any new risks that may be found during the course of the study?

You will be promptly notified if, during the conduct of this research study, any new information develops which may cause you to change your mind about continuing to participate.

Will there be any associated costs with this study?

There will be no costs to participants within this study.

Will I be paid if I take part in this research study?

There is no monetary compensation for study participants.

Who will know about my participation in this research study?

Any information about you obtained from this research will be kept as confidential (private) as possible. This identifiable information will be made available to members of the research team and their staff 
for an indefinite period of time. All records related to your involvement in this research study will be kept private. Your identity on these records will be indicated by a case number rather than by your name, and the information linking these case numbers with your identity will be kept separate from the research records.

At the end of this study, any records that personally identify you will remain stored in locked files and will be kept for a minimum of seven years. Your identity will not be revealed in any description or publications of this research. Data may be shared with others, but it will be shared without identifiers.

In unusual cases, your research records may be released in response to an order from a court of law. It is also possible that authorized representatives from the University of Pittsburgh Research Conduct and Compliance Office may review your data for the purpose of monitoring the conduct of this study. Also, if the investigators learn that you or someone with whom you are involved is in serious danger or potential harm, they will need to inform the appropriate agencies, as required by Pennsylvania law.

\section{Is my participation in this study voluntary?}

You may withdraw your authorization to allow the research team to review your medical records at any time. If you withdraw consent, you will no longer be permitted to participate in this study. If you decide you no longer wish to continue to participate after signing this consent form, you should contact Kristen Qutub (908-839-8794). If you choose not to sign this consent form, you will not be enrolled in this study. Your decision will not affect your relationship with the University of Pittsburgh, the University of Pittsburgh Medical Center, or a health care insurance provider; nor will you lose any benefits that you might be eligible for because of what you decide.

Any information collected prior to the date that you withdraw your consent may continue to be used by the investigators for the purposes described above. You may be withdrawn from the study at any time by the investigators: for example, if you were found to meet any of the study criteria that would exclude you from participating. 


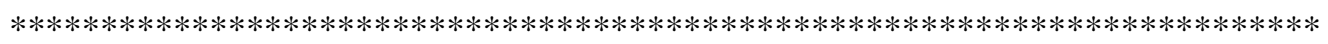

\section{VOLUNTARY CONSENT}

The above information has been explained to me and all of my current questions have been answered. I understand that I am encouraged to ask questions about any aspect of this research study during the course of this study, and that such future questions will be answered by the primary investigator listed on the first page of this consent document at the telephone number given.

I understand that I may contact the Human Subjects Protection Advocate of the IRB Office, University of Pittsburgh (1-866-212-2668) to discuss problems, concerns, and questions; obtain information; offer input; or discuss situations that have occurred during my participation.

By signing this form, I agree to participate in this research study. A copy of this consent form will be given to me.

Participant’s Signature

Date

Printed Name of Participant

\section{CERTIFICATION of INFORMED CONSENT}

I certify that I have explained the nature and purpose of this research study to the above-named individual(s), and I have discussed the potential benefits and possible risks of study participation. Any 
questions the individual(s) have about this study have been answered, and we will always be available to address future questions as they arise.”

Printed Name of Person Obtaining Consent

Signature of Person Obtaining Consent
Role in Research Study

Date 


\section{DEMOGRAPHIC SURVEY}

\section{DEMOGRAPHIC DATA}

\section{CAREGIVER}

1. Age

2. Male Female

3. Are you currently working?

Y Yes

a) Current Occupation:

b) Full time __ Part time __ Per Diem

_ No

b) Date stopped working:

4. Have you had to cut back on your work hours as a result of caregiving duties?

_ No Not working when patient was diagnosed

- Yes

\section{Education}

_ Graduate degree

__ College degree

__ Some college

High school graduate 
__ Did not complete high school

6. Current household annual income/year

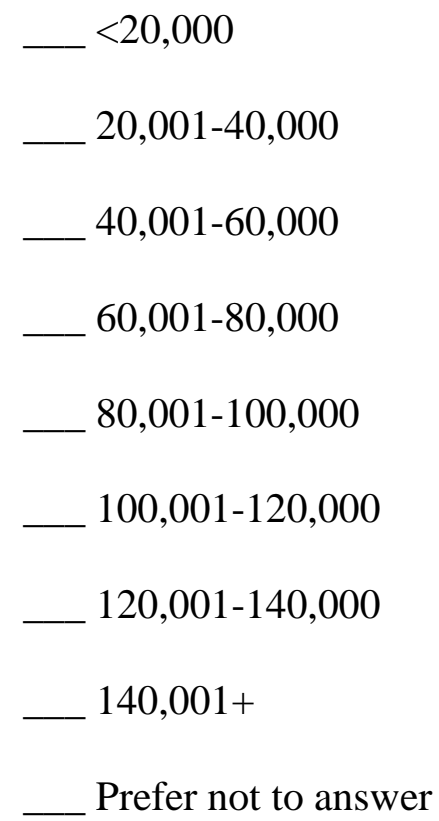

7. Overall, how adequate are your finances/insurance in covering health care costs?

___ Adequate (not a financial burden)

__ Inadequate (small financial burden)

__ Inadequate (significant burden)

\section{Current Living Arrangement}

With Spouse or Partner only

_ _ With spouse/partner and children

__ With children only

__ With other family

Roommate/Friends

Alone

Other 
9. As a caregiver, about how many hours each day are you with the patient (helping patient, socializing, or simply being nearby to help, if called upon)?

$$
\text { \# of hours per day: }
$$

10. As a caregiver, about how many hours each day are you with the patient providing hands-on help (feeding patient, suctioning, helping patient communicate)?

$$
\text { \# of hours per day: }
$$

11. Does anyone else provide regular hands-on care each week?

- No

_ Y Yes If yes, how many hours do you receive help per day?

12. Do you feel you receive adequate support from family and friends on a regular basis?

— No

_ Yes

\section{Current Religious Identification}

_ Catholic

_ Protestant

__ Other Christian

__ Jewish

_ Muslim

_ Hindu

__ Buddhist

_ Agnostic/Spiritual

Atheist/No religion

Other 
14. How religious do you consider yourself?

$\ldots$ Very religious
Religious
A little religious
Not religious

15. Does religious play a daily role in your life?

No
Yes




\section{BIBLIOGRAPHY}

ALS Association, ALSA. (2010). Amyotrophic Lateral Sclerosis.

Beck, A. T., \& Steer, R. A. (1984). Internal consistencies of the original and revised Beck Depression Inventory. J Clin Psychol, 40(6), 1365-1367.

Beck, A. T., Ward, C. H., Mendelson, M., Mock, J., \& Erbaugh, J. (1961). An inventory for measuring depression. Arch Gen Psychiatry, 4, 561-571.

Bedard, M., Molloy, D. W., Squire, L., Dubois, S., Lever, J. A., \& O'Donnell, M. (2001). The Zarit Burden Interview: a new short version and screening version. Gerontologist, 41(5), 652-657.

Belden, Russonello \& Stewart. (2004). Caregiving in the U.S. In MetLife (Ed.), National Alliance for Caregiving \& AARP.

Calvo, A., Moglia, C., Ilardi, A., Cammarosano, S., Gallo, S., Canosa, A., Mastro, E., Montuschi, A., \& Chiò, A. (2011). Religiousness is positively associated with quality of life of ALS caregivers. Amyotroph Lateral Sclerosis, 12, 168-171.

CDC. (2010). Current Depression Among Adults - United States, 2006 and 2008. MMWR, 38, 1229-1235.

Chio, A., Gauthier, A., Calvo, A., Ghiglione, P., Mutani, R. (2005). Caregiver burden and patients' perception of being a burden in ALS. Neurology, 64, 1780-1782.

Coughlin, J. (2010). Estimating the Impact of Caregiving and Employment on Well-Being. Outcomes \& Insights in Health Management, 2(1).

Department of Mental Health, CA. (2005). A 20-year partnership in caring California Caregiver Resource Centers.

Ganzini, L., Johnston, W., McFarland, B., Tolle, S., Lee, M. (1998). Attitudes of patients with amyotrophic lateral sclerosis and their care givers toward assisted suicide. The New England Journal of Medicine, 339, 967-973.

Gauthier, A., Vignola, A., Calvo, A., Cavallo, E., Moglia, C., Sellitti, L., Mutani, R., Chio, A. (2007). A longitudinal study on quality of life and depression in ALS patient-caregiver couples. Neurology, 68, 923-926.

Goldstein, L. Atkins, S. Landau, R. Brown, P.N. Leigh. (2006). Predictors of psychological distress in carers of people with amyotrophic lateral sclerosis: a longitudinal study. Psychological Medicine, 36, 865-875.

Health and Human Services, Department. (1998). Informal Caregiving: Compassion in Action. Kennedy, J. \& Walls, C. (1997). A National Profile of IntraHousehold ADL/IADL Assistants: Population Estimates From the 1992 and 1993 Surveys of Income and Program Participation. Department of Community Health, University of Illinois at Urbana Champaign. 
Lillo, P., Mioshi, E., and Hodges, J. (2012). Caregiver burden in amyotrophic lateral sclerosis is more dependent on patients' behavioral changes than physical disability: a comparative study. BMC Neurology, 12, 156.

Mayo Clinic, staff. (2011). Amyotrophic Lateral Sclerosis. Retrieved April 3, 2013

Metropolitan Life, Insurance Company. (2010). Working Caregivers and Employer Health Care Costs. University of Pittsburgh Institute on Aging, Case Study.

Mockford, C., Jenkinson, C., \& Fitzpatrick, R. (2006). A Review: carers, MND and service provision. Amyotroph Lateral Scler, 7(3), 132-141. doi: 10.1080/14660820600601028

National Institute of Health, NIH. (2013). Amyotrophic Lateral Sclerosis (ALS) Fact sheet. Retrieved April 3, 2013.

National Institute of Neurological Disorders and Stroke, NINDS. (2012). Amyotrophic Lateral Sclerosis (ALS) Fact Sheet. 2013.

Oyebode, J. R., Smith, H. J., \& Morrison, K. (2013). The personal experience of partners of individuals with motor neuron disease. Amyotroph Lateral Scler Frontotemporal Degener, 14(1), 39-43.

Pagnini, F., Rossi, G., Lunetta, C., Banfi, P., Castelnuovo, G., Corbo, M., Molinari, E. (2010). Burden, depression, and anxiety in caregivers of people with amyotrphic lateral sclerosis. Psychology, Health, \& Medicine, 15, 685-693.

Rabkin, J., Albert, S., Rowland, L., \& Mitsumoto, H. (2009). How common is depression among ALS caregivers? A longitudinal study. Amyotroph Lateral Sclerosis, 10, 448-455.

Ray, R. and Street, A. (2005). Who's there and who cares: age as an indicator of social support networks for caregivers among people living with motor neurone disease. Health and Social Care in the Community, 13(542-552).

Shultz, R., O'Brien, A., Bookwala, J., \& Fleissner, K. (1995). Psychiatric and Physical Morbidity Effects of Dementia Caregiving: Prevalence, Correlates, and Causes. The Gerontologist, 35, 771-791.

Trail, M., Nelson, N., Van, J., Appel, S., Lai, E. . (2003). A study comparing patients with amyotrophic lateral sclerosis and their caregivers on measures of quality of life, depression, and their attitudes toward treatment options. Journal of the Neurological Sciences, 209, 79-85.

Wagner, D.L. (1997). Long Distance Caregiving for Older Adults. Healthcare and Aging, National Council on the Aging. 